University of Wollongong

Research Online

Faculty of Engineering and Information

Faculty of Engineering and Information

Sciences - Papers: Part A

Sciences

$1-1-2013$

Ionization cross section data of nitrogen, methane, and propane for light ions and electrons and their suitability for use in track structure simulations

Marion Bug

University of Wollongong, mb355@uowmail.edu.au

Elisabetta Gargioni

Universit Atsklinikum Hamburg-Eppendorf

Heidi Nettelbeck

Physikalisch-Technische Bundesanstalt (PTB), hn06@uow.edu.au

Woon Yong Baek

Physikalisch-Technische Bundesanstalt Germany

Gerhard Hilgers

Physikalisch-Technische Bundesanstalt (PTB)

See next page for additional authors

Follow this and additional works at: https://ro.uow.edu.au/eispapers

Part of the Engineering Commons, and the Science and Technology Studies Commons

Research Online is the open access institutional repository for the University of Wollongong. For further information contact the UOW Library: research-pubs@uow.edu.au 


\title{
Ionization cross section data of nitrogen, methane, and propane for light ions and electrons and their suitability for use in track structure simulations
}

\begin{abstract}
Track structure Monte Carlo simulations are frequently applied in micro- and nanodosimetry to calculate the radiation transport in detail. The use of a well-validated set of cross section data in such simulation codes ensures accurate calculations of transport parameters, such as ionization yields. These cross section data are, however, scarce and often discrepant when measured by different groups. This work surveys literature data on ionization and charge-transfer cross sections of nitrogen, methane, and propane for electrons, protons, and helium particles, focusing on the energy range between $100 \mathrm{keV}$ and $20 \mathrm{MeV}$. Based on the evaluated data, different models for the parametrization of the cross section data are implemented in the code PTRA, developed for simulating proton and alpha particle transport in an ioncounting nanodosimeter. The suitability of the cross section data is investigated by comparing the calculated mean ionization cluster size and energy loss with experimental results in either nitrogen or propane. For protons, generally good agreement between measured and simulated data is found when the Rudd model is used in PTRA. For alpha particles, however, a considerable influence of different parametrizations of cross sections for ionization and charge transfer is observed. The PTRA code using the charge-transfer data is, nevertheless, successfully benchmarked by the experimental data for the calculation of nanodosimetric quantities, but remaining discrepancies still have to be further investigated (up to $13 \%$ lower energy loss and $19 \%$ lower mean ionization cluster size than in the experiment). A continuation of this work should investigate data for the energy loss per interaction as well as differential cross section data of nitrogen and propane. Interpolation models for ionization and charge-transfer data are proposed. The Barkas model, frequently used for a determination of the effective charge in the ionization cross section, significantly underestimates both the energy loss (by up to 19\%) and the mean ionization cluster size (up to $65 \%$ ) for alpha particles. It is, therefore, not recommended for particle-track simulations.
\end{abstract}

\section{Keywords}

section, data, nitrogen, methane, propane, light, ions, electrons, ionization, their, cross, suitability, track, structure, simulations

\section{Disciplines}

Engineering | Science and Technology Studies

\section{Publication Details}

Bug, M., Gargioni, E., Nettelbeck, H., Baek, W., Hilgers, G., Rozenfeld, A. \& Rabus, H. (2013). Ionization cross section data of nitrogen, methane, and propane for light ions and electrons and their suitability for use in track structure simulations. Physical Review E: Statistical, Nonlinear, and Soft Matter Physics, 88 (4), 043308-1-043308-21.

\section{Authors}

Marion Bug, Elisabetta Gargioni, Heidi Nettelbeck, Woon Yong Baek, Gerhard Hilgers, Anatoly B.

Rosenfeld, and Hans Rabus 


\title{
Ionization cross section data of nitrogen, methane, and propane for light ions and electrons and their suitability for use in track structure simulations
}

\author{
Marion U. Bug,,${ }^{1,2}$ Elisabetta Gargioni, ${ }^{3}$ Heidi Nettelbeck, ${ }^{1}$ Woon Yong Baek, ${ }^{1}$ \\ Gerhard Hilgers, ${ }^{1}$ Anatoly B. Rosenfeld, ${ }^{2}$ and Hans Rabus ${ }^{1}$ \\ ${ }^{1}$ Physikalisch-Technische Bundesanstalt (PTB), Bundesallee 100, 38116 Braunschweig, Germany \\ ${ }^{2}$ Centre for Medical Radiation Physics (CMRP), University of Wollongong, Wollongong, New South Wales 2522, Australia \\ ${ }^{3}$ University Medical Center Hamburg-Eppendorf, Martinistrasse 52, 20246 Hamburg, Germany
}

(Received 8 August 2013; published 29 October 2013)

\begin{abstract}
Track structure Monte Carlo simulations are frequently applied in micro- and nanodosimetry to calculate the radiation transport in detail. The use of a well-validated set of cross section data in such simulation codes ensures accurate calculations of transport parameters, such as ionization yields. These cross section data are, however, scarce and often discrepant when measured by different groups. This work surveys literature data on ionization and charge-transfer cross sections of nitrogen, methane, and propane for electrons, protons, and helium particles, focusing on the energy range between $100 \mathrm{keV}$ and $20 \mathrm{MeV}$. Based on the evaluated data, different models for the parametrization of the cross section data are implemented in the code PTRA, developed for simulating proton and alpha particle transport in an ion-counting nanodosimeter. The suitability of the cross section data is investigated by comparing the calculated mean ionization cluster size and energy loss with experimental results in either nitrogen or propane. For protons, generally good agreement between measured and simulated data is found when the Rudd model is used in PTRA. For alpha particles, however, a considerable influence of different parametrizations of cross sections for ionization and charge transfer is observed. The PTRA code using the charge-transfer data is, nevertheless, successfully benchmarked by the experimental data for the calculation of nanodosimetric quantities, but remaining discrepancies still have to be further investigated (up to $13 \%$ lower energy loss and $19 \%$ lower mean ionization cluster size than in the experiment). A continuation of this work should investigate data for the energy loss per interaction as well as differential cross section data of nitrogen and propane. Interpolation models for ionization and charge-transfer data are proposed. The Barkas model, frequently used for a determination of the effective charge in the ionization cross section, significantly underestimates both the energy loss (by up to 19\%) and the mean ionization cluster size (up to 65\%) for alpha particles. It is, therefore, not recommended for particle-track simulations.
\end{abstract}

DOI: 10.1103/PhysRevE.88.043308

PACS number(s): 07.05.Tp, 87.53.Bn

\section{INTRODUCTION}

Micro- and nanodosimetric approaches have been under development for several years [1-6] as a means to characterize the track structure of ionizing radiation. This characterization is particularly important for an estimation of initial radiationinduced biological effects on the microscopic scale. At such small dimensions, the stochastic nature of radiation interactions with the medium, manifesting in the track structure, has to be considered in detail. The particle track consists of a sequence of single interactions of the primary particle with the traversed medium and the interactions of secondary particles, which are produced during ionization processes and subsequently propagate in the medium. A description of the track structure is particularly important for densely ionizing radiation, such as ions (with an energy of their stopping power maximum) or low-energy secondary electrons (below about $1 \mathrm{keV})$. These particles deposit a large amount of their energy within volumes of a few micrometers or even nanometers and therefore lead to significant damage of the microscopic structure of matter. In the case of the DNA, this may lead to carcinogenesis or cell death. Therefore, track structure quantities describing the density of interactions, which potentially produce lesions to the DNA on the microscopic scale, need to be accurately determined.

Track structure quantities are experimentally investigated in micro- and nanodosimetry using detectors filled with a low density gas $[1,7,8]$. Such experiments have, in fact, proven to effectively model parameters related to the track structure in microscopic compartments of human cells for particles of different type and energy (defining the radiation quality) [8]. While microdosimeters often use tissue-equivalent gases, consisting of a mixture of propane, nitrogen, and carbon dioxide, nanodosimeters are usually operated with either nitrogen or propane gas $[1,2,7]$.

In addition to experiments, Monte Carlo track structure simulations are important for characterizing particle tracks in condensed media, such as human cells. In such media, track structure quantities cannot be experimentally determined, owing to the limitations of current detector technology. Particle-track simulation codes can, however, be benchmarked with measured data by modeling a micro- or nanodosimetric experiment, which is performed in millimeter-sized volumes of low-pressure gas $[7,8]$. To simulate the particle track, the history of an incident projectile is followed interaction by interaction through the medium $[3,8,9]$. For such detailed simulations, the cross sections for the physical interactions of the incident particles with the molecules comprising the medium are essential input data.

The most important cross sections for nano- and microdosimetric applications are those for impact ionization since these dosimeters measure the number of ionized target molecules or the energy deposited by ionizations. Despite the frequent use of propane in dosimetry, a survey of the literature indicates that ionization or charge-transfer cross sections for light ions 
in this medium have rarely been measured. For example, measured data on proton or alpha particle impact-ionization cross sections of propane are still missing. However, propane data can be scaled from those of methane as explained in Sec. IV D. For nitrogen, on the other hand, a large amount of interaction cross section data for protons and electrons are available as well as a few data for helium particles. However, some of these data appear inconsistent in overlapping energy ranges.

The aim of this work is to recommend cross section data sets of nitrogen and propane for light ions (i.e., protons and helium particles) and electrons, which can be implemented in track structure codes that simulate nano- and microdosimetry experiments. For this purpose, data on impact-ionization cross sections of nitrogen, propane, and methane available in the literature are surveyed for electrons, as well as for protons and helium particles of different charge states. Charge-transfer cross sections for helium particles are reviewed as well. Methods to provide a complete data set for an implementation in track structure simulations are investigated, using model functions for interpolation. Simulated nanodosimetric quantities are compared with experimental results to benchmark the simulations. Also, the influence of different models used for parameterizing the interaction cross sections on calculated quantities is assessed.

Section II briefly introduces the nanodosimetric approach applied in this work. Details on the track structure simulation by means of the PTRA code are presented in Sec. III. Review, analysis, and modeling of the cross section data are discussed in Sec. IV. In Sec. V, simulation results are compared to experimental data.

\section{BASIC NANODOSIMETRIC QUANTITIES}

The nanodosimetric approach applied in this work is based on the evaluation of the ionization cluster size, which is defined as the number of ionizations produced by a passage of a single incident particle within a specified target volume. Ionizations of the primary ions and secondary electrons occur at random positions along the track and subsequent ionizations are spatially separated, on average, by the ionization mean free path. Therefore, the ionization cluster size is a stochastic quantity and can be characterized by a probability distribution. The ionization mean free path is a function of the radiation quality $Q$ so that the probability distribution $P(v \mid Q)$ of ionization cluster size $v$ is a quantity characterizing the track structure of a specific radiation quality. $P(\nu \mid Q)$ is subject to the normalization condition

$$
\sum_{\nu=0}^{\infty} P(\nu \mid Q)=1
$$

Further characteristic quantities describing the track structure for a given radiation quality can be derived from momenta of $P(v \mid Q)$. For example, the mean ionization cluster size $M_{1}$ is defined by its first moment,

$$
M_{1}=\sum_{\nu=0}^{\infty} \nu P(\nu \mid Q) .
$$

\section{PTRA TRACK STRUCTURE SIMULATIONS}

The Physikalisch-Technische Bundesanstalt (PTB) track structure code "PTRA" is dedicated for applications in nanodosimetry $[4,8]$. This code also models the experimental setup of the ion-counting nanodosimeter which has been developed by the PTB and Weizmann Institute of Science (WIS) and has been comprehensively described in [7]. In brief, the nanodosimeter operates with either nitrogen or propane at a pressure on the order of $120 \mathrm{~Pa}$. It was used at the PTB ion accelerator facilities to measure track structure parameters of protons and alpha particles of energies between $100 \mathrm{keV}$ and $20 \mathrm{MeV}$. Incident ions enter a gas-filled chamber through a Mylar window. They subsequently traverse $230 \mathrm{~mm}$ of gas before reaching the so-called sensitive volume of the nanodosimeter, which is defined by an electrical field. It is approximately cylindrical in shape with a height of $50 \mathrm{~mm}$ and a diameter of about $1 \mathrm{~mm}$. Gas ions created inside the sensitive volume by the passage of an incident particle drift along an appropriate electric field and are then extracted through an aperture and counted. The spatial variation of the extraction efficiency of the gas ions is taken into account in the simulations. After traversing the sensitive volume, the residual energy of the primary ion is recorded by a silicon detector, positioned $75 \mathrm{~mm}$ downstream of the sensitive volume.

In the past years, PTRA was used to simulate the transport of protons and alpha particles with energies above $1 \mathrm{MeV} / \mathrm{u}$, for which charge-transfer processes can be neglected. Simulations with the PTRA code have shown to reproduce well the probability distribution of ionization cluster sizes produced by $4.6-\mathrm{MeV}$ alpha particles in volumes of nitrogen, measured with another nanodosimeter known as the Jet Counter [4]. Furthermore, experimental cluster size distributions in propane obtained with an ion-counting nanodosimeter, similar to that of PTB/WIS, for 4.3-MeV alpha particles and protons of energies above $7 \mathrm{MeV}$ also showed good agreement with calculated data [7]. Even simulated cluster size distributions for protons of energies between 0.4 and $3.5 \mathrm{MeV}$ in propane are in overall good agreement with the data measured in the PTB/WIS ion-counting nanodosimeter [10].

In the simulations, the ions are started behind the Mylar window and the history of each primary ion, traversing the interaction chamber, is calculated until it reaches the detector plane. ${ }^{1}$ Secondary electrons are transported until their energy falls below the ionization threshold of the gas molecules (only the yield of ionization events is of interest in this work). Particle tracks are calculated in PTRA by taking into account the processes described in the following. Elastic scattering of electrons is included, where the direction of an electron is changed according to the differential elastic scattering cross section and without any deposition of energy. Impact ionization by an ion or electron leads to the emission of a secondary electron, which is subsequently transported. In the case of an electronic excitation, the projectile transfers some energy to the target molecule, resulting in the excitation of an electron to a higher energy level. In the simulations, a

\footnotetext{
${ }^{1}$ Versions PTra-n2-1302 and PTra-c3h8-1302 were used for simulations in nitrogen and propane, respectively.
} 
potential change in direction of the projectile by excitation processes was neglected. Moreover, only a minor fraction of the energy loss by an incident particle originates from excitation processes, since the excitation cross section is significantly lower than that for ionization and, on average, less energy is transferred in an excitation event $[11,12]$. Therefore, the influence of electronic excitations of gas molecules by ions on the ionization cluster size is negligible and these processes are not further discussed in this work. The total and differential cross section data for the processes described above, previously implemented in PTRA, are summarized in Refs. [4,8,13].

For an accurate transport simulation of low-energy protons (below $1 \mathrm{MeV}$ ) and alpha particles (below $4 \mathrm{MeV}$ ) through a medium, it is necessary to consider charge-transfer processes of the projectile, which become increasingly important as the particle energy decreases [14]. Charge-transfer processes for protons in the energy range down to $100 \mathrm{keV}$ may not have a considerable effect on the proton's track structure, as is discussed in Sec. IV C; therefore, charge-transfer processes are only described for alpha particles in the following. In a charge-transfer process, alpha particles can ionize the target molecule by capturing one or two of the target's electrons and then continue their passage as singly charged helium ions, $\mathrm{He}^{+}$, or neutral helium atoms, $\mathrm{He}^{0} . \mathrm{A} \mathrm{He}^{+}$ion can, in turn, capture another electron to become a neutral $\mathrm{He}^{0}$ atom. Since the probability for a $\mathrm{He}^{0}$ atom to capture an electron is almost negligible [15], this process is not further considered here. The cross sections for ionization of gas molecules by helium particles of lower charge states are significantly lower than for alpha particles. Both $\mathrm{He}^{+}$and $\mathrm{He}^{0}$ projectiles can also experience electron loss, where one or two electrons are stripped off the projectile. Such electrons will travel through the medium with a well-defined probability of ionization, depending on their energy. In summary, the charge-transfer processes considered for simulating the transport of low-energy alpha particles are: the single and double electron-capture cross sections for $\mathrm{He}^{2+}$ $\left(\sigma_{21}\right.$ and $\left.\sigma_{20}\right)$; the single electron-capture and electron-loss processes for $\mathrm{He}^{+}\left(\sigma_{10}\right.$ and $\left.\sigma_{12}\right)$; and the single and double electron-loss cross sections for $\mathrm{He}^{0}\left(\sigma_{01}\right.$ and $\left.\sigma_{02}\right)$.

An incident (monoenergetic) helium beam reaches a state of charge equilibrium after a few interactions. In this case, the probability for a specific charge state is determined by the equilibrium condition that the rate of projectiles gaining a specific charge state is equal to the rate of those losing this charge state. More specifically, for an ensemble of helium particles, a particular electron-capture process $\sigma_{i j}$ is always balanced by the corresponding electron-loss process $\sigma_{j i}$, such that

$$
f_{i} \sigma_{i j}=f_{j} \sigma_{j i},
$$

where $f_{i}$ and $f_{j}$ are the equilibrium fractions of projectiles present in a specific charge state $i$ and $j$, respectively, subject to

$$
\sum_{i=0}^{i_{\max }} f_{i}=1
$$

where $i_{\max }=2$ for helium projectiles. Therefore, the equilibrium charge distribution is independent of the initial charge distribution in the beam and is only a function of the projectile energy.

Charge-transfer processes were included in the track structure simulation using two different approaches. In the first approach, the cross section data of these processes were directly incorporated in the random sampling procedure, where each interaction type was determined by taking into account the charge state of the helium projectile (in particular, the cross section data for ionization and charge transfer as well as total scattering cross sections). In the case of charge transfer, the charge of the helium particle was modified accordingly, before sampling the path length to the next point of interaction. In this, several assumptions were made. For example, in an electron-capture process, the energy change of the projectile is neglected. This energy change would arise from the difference in the binding energy of the electron initially bound in the target molecule and the binding energy in the final bound state in the projectile on the one hand and the energy required to accelerate the captured electron to the velocity of the projectile on the other hand. Furthermore, the energy required to remove the electron from the projectile in an electron-loss process was also neglected. This is reasonable as the binding energies are small compared to the projectile energies and the electron mass is much smaller than the mass of the projectile ion. In this case, the alteration in projectile energy due to the above mentioned effects would not lead to a significant change in the projectile's interaction cross sections. Finally, free electrons produced by electron-loss processes of the projectile were further transported through the medium, with their initial velocity assumed to be equal to that of the projectile with a momentum in the forward direction. The second approach used the equilibrium fractions of the charge states [according to Eq. (3)] to calculate an effective ionization cross section, which per se takes the above listed assumptions into account (see Sec. IV G).

\section{IONIZATION AND CHARGE-TRANSFER CROSS SECTIONS}

This section provides a literature survey of the total ionization and charge-transfer cross sections of nitrogen, methane, and propane for interactions with electrons, protons, and helium particles. A review of semiempirical models is also included and recommendations on cross section data, suitable for an application in track structure simulations, are given. The literature review is not exhaustive for all particle types and energies, but rather focuses on data covering the energy range of interest for track structure simulations of protons and alpha particles in nanodosimetry (i.e., $100 \mathrm{keV}$ to $20 \mathrm{MeV}$ ).

\section{A. Electrons in nitrogen}

A large number of cross section data exist for the interaction of electrons with nitrogen. Itikawa [16] comprehensively surveyed the literature and recommended a cross section data set. The recommended data agree within experimental uncertainties with probably the most referenced cross section data of Rapp and Englander-Golden [17] (Fig. 1). The binaryencounter-Bethe (BEB) model [19] (see Appendix A) predicts the measured data down to the maximum of the cross section at 


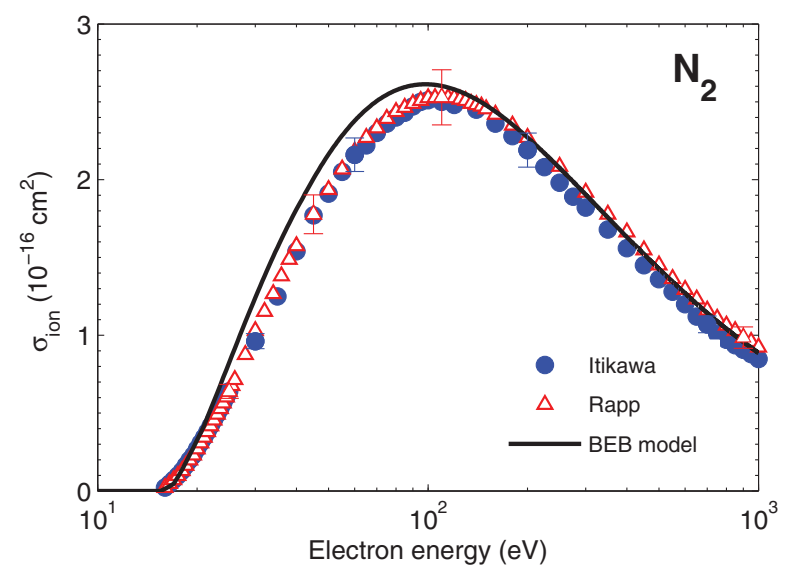

FIG. 1. (Color online) Electron-impact-ionization cross sections $\sigma_{\text {ion }}$ of nitrogen recommended by Itikawa [16], measured by Rapp and Englander-Golden [17], and determined using the BEB model [18].

an energy of $100 \mathrm{eV}$ within a stated experimental uncertainty of 5\%. For lower energies, the deviation is as much as $30 \%$ and occurs at an energy of about $30 \mathrm{eV}$. This deviation might be due to the approximation of differential oscillator strengths in the model. Nevertheless, the PTRA code uses the BEB model with the molecular orbital data from Hwang et al. [18] for describing the electron ionization processes in nitrogen, because this model provides also partial ionization cross sections for the subshells (needed to determine the projectile's enery loss).

\section{B. Electrons in propane and methane}

Electron interaction cross sections have been experimentally and theoretically determined for both propane and methane by several groups (e.g., those referenced in Table I). Figure 2 shows a selection of ionization cross sections for both molecules. In the case of methane, the BEB model in combination with the molecular orbital data of the NIST database $[18,20]$ reproduces the measured data of Durić et al. [21] and Schram et al. [22] within the experimental uncertainties. The cross sections measured by Nishimura et al. [23], on the other hand, are systematically larger (up to $20 \%$ in the energy range between $100 \mathrm{eV}$ and $2 \mathrm{keV}$ ) than those predicted by the

TABLE I. Electron-impact-ionization cross section data of methane and propane and model functions for an interpolation of cross sections. Data are in $\mathrm{eV}$.

\begin{tabular}{lcc}
\hline \hline & Experimental & \\
Durić et al. [21] & $\mathrm{CH}_{4}, \mathrm{C}_{3} \mathrm{H}_{8}$ & $12-240$ \\
Grill et al. [24] & $\mathrm{CH}_{4}$ & $15-950$ \\
Nishimura et al. [23] & $\mathrm{CH}_{4}, \mathrm{C}_{3} \mathrm{H}_{8}$ & $15-3000$ \\
Schram et al. [22] & $\mathrm{CH}_{4}, \mathrm{C}_{3} \mathrm{H}_{8}$ & $600-12000$ \\
& $\mathrm{Theoretical}$ & \\
De Souza et al. [25] & $\mathrm{CH}_{4}$ & $2-500$ \\
Vinodkumar et al. [26] & $\mathrm{CH}_{4}$ & $15-2000$ \\
& $\mathrm{Models}$ & \\
BEB model [19] & & \\
$\quad$ (see Appendix A) & & \\
Chouki model [27] & & \\
(see Appendix B) & & \\
\hline \hline
\end{tabular}

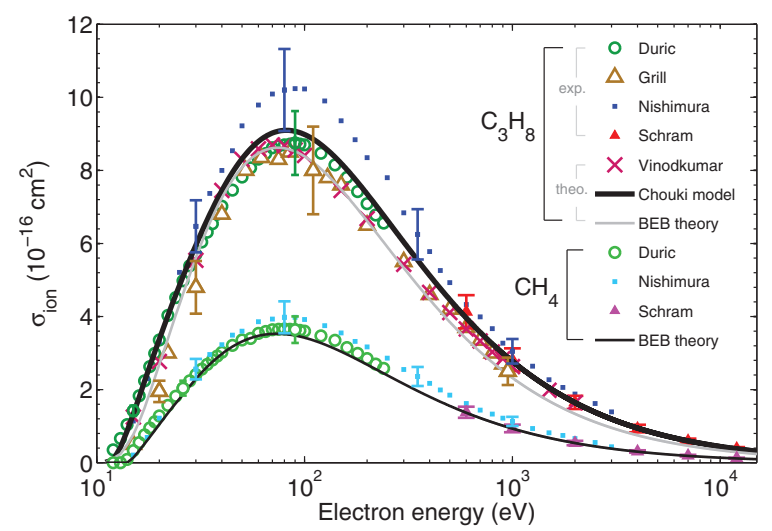

FIG. 2. (Color online) Electron-impact-ionization cross sections $\sigma_{\text {ion }}$ of methane and propane (references are listed in Table I). Error bars are only shown for selected data points to improve readability.

BEB model, although an acceptable agreement is reached at lower energies when considering experimental uncertainties. All three groups determined the total ionization cross section by measuring the current originating from the collection of positive charges, produced by the passage of an electron beam.

For propane, larger deviations between the available cross section data sets for electrons are observed (Fig. 2). Again, the data of Nishimura are systematically higher (up to 25\%) than those determined by Durić et al. [21], especially in the intermediate energy range between $50 \mathrm{eV}$ and $1 \mathrm{keV}$. This deviation exceeds the reported experimental uncertainties, which were between $10 \%$ and $15 \%$ for all experimental data. On the contrary, the cross section data of Grill et al. [24] are systematically lower than those of Durić et al. (up to $65 \%$ below $30 \mathrm{eV}$ ), although the discrepancy above $30 \mathrm{eV}$ (approximately 10\%) can be considered negligible within the overall uncertainty. Grill et al. determined partial ionization cross sections by measuring the number of positively charged molecular fragments, produced by the passage of an electron beam, by means of a mass spectrometer. For higher electron energies (above $600 \mathrm{eV}$ ), the cross section data measured by Schram et al. [22] agree with the data of Grill et al. and Nishimura et al. within about $10 \%$.

The data of Durić et al. are supported by the inelastic cross sections of de Souza et al. [25], calculated by an ab initio approach, which are not shown in Fig. 2 in the interest of readability. However, for energies above $400 \mathrm{eV}$ de Souza's predictions fall even below the predictions of the BEB model. Unfortunately, their data are only provided for energies up to $500 \mathrm{eV}$, thus not allowing the assessment of the high-energy behavior. Also the theoretical cross section data of Vinodkumar et al. [26], determined by the spherical complex optical potential approach, support the data of Durić et al. for energies below $80 \mathrm{eV}$, but better reproduce those of Grill et al. for energies between the maximum of the electron cross section at about $80 \mathrm{eV}$ and $1 \mathrm{keV}$. In this energy range, the two data sets agree within $10 \%$ to $15 \%$, which is within the stated experimental uncertainties. Above $1 \mathrm{keV}$, the cross sections of Vinodkumar et al. are in good agreement with those measured by Schram et al. (well within the $11 \%$ experimental uncertainty). 
The BEB model using the molecular orbital data for propane [20], agrees well with the experimental data and those calculated by Vinodkumar et al. up to an electron energy of about $400 \mathrm{eV}$. Above this energy, however, the data obtained from the BEB model are lower than those of Schram et al. and Vinodkumar et al. (about $20 \%$ at $1 \mathrm{keV}$ ) and this deviation even increases with increasing energy (25\% at $12 \mathrm{keV})$. This observation seems to be in contrast to the good agreement of the BEB model with Schram's measured data for methane over the same energy range, but this tendency has also been observed by Hwang et al. [18]. Their comparison of the BEB model with experimental ionization cross section data for different hydrocarbon molecules indicated larger deviations at higher energies for alkanes of higher order. It is not clear whether this deviation arises from systematical uncertainties of the measurements or from deficiencies of the model. For this reason, the parametrization of electron ionization cross sections of propane for the use in PTRA was obtained by calculating the mean value of the measured cross section data of Grill et al., Schram et al., Durić et al., and Nishimura et al. The Chouki model [27] was then used to fit this data set. This model, together with the parameters for propane, is summarized in Appendix B. In the high-energy range, the Chouki model gives about $20 \%$ larger cross section values than the BEB model (Fig. 2).

\section{Protons in nitrogen}

Total ionization cross sections of nitrogen for protons in the energy range of interest in this work (between $100 \mathrm{keV}$ and $20 \mathrm{MeV}$ ) were measured by several groups (see Table II) and a comprehensive survey and evaluation of literature data has been published by Rudd et al. in 1985 [28].

Figure 3 illustrates that the data of energies above $80 \mathrm{keV}$ are generally in good agreement within the experimental uncertainties (which were between $10 \%$ and $25 \%$ ). The measurements of Knudsen et al. [31] differ by less than $10 \%$ from those of the other groups (no uncertainty was provided), with the exception of the two lowest energy data points. Knudsen et al. determined the single-ionization cross sections by measuring the yield of positively charged fragments produced after the passage of a proton through a low-density gas. This yield was then corrected for the fraction of hydrogen atoms produced

TABLE II. Impact-ionization cross section data and equilibrium fractions for hydrogen projectiles in nitrogen and model functions for an interpolation of cross sections. Data in $\mathrm{keV}$.

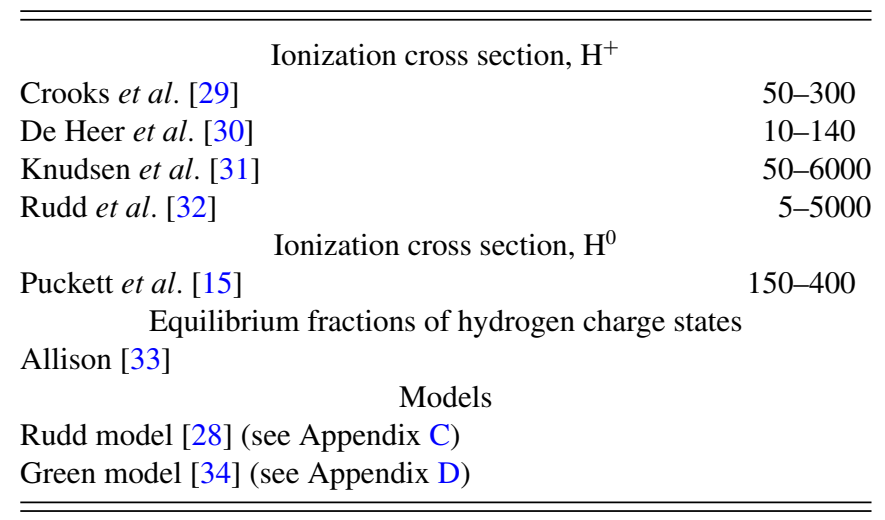

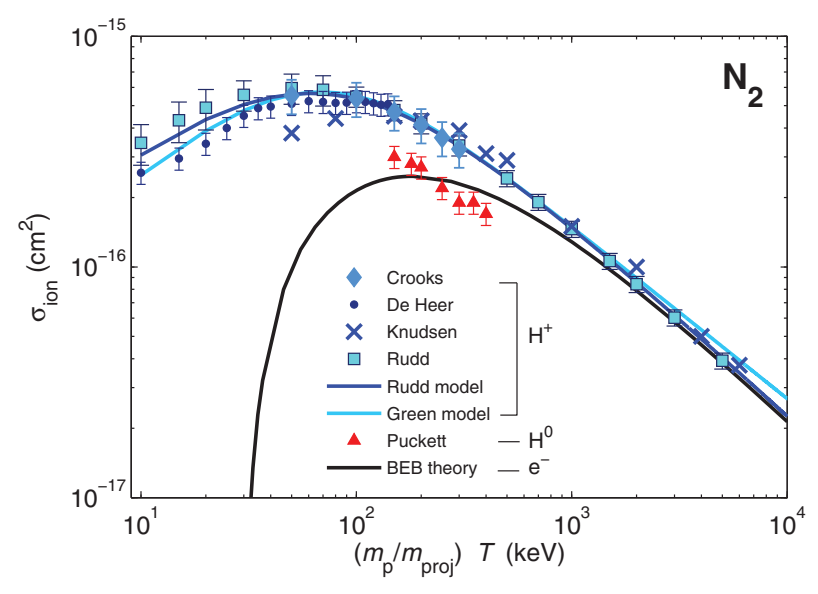

FIG. 3. (Color online) Ionization cross sections $\sigma_{\text {ion }}$ of nitrogen for protons and electrons. Shown are measured ionization cross section data for protons and neutral hydrogen atoms (symbols) as well as results of semiempirical models (lines) (see Table II for references). Electron ionization cross sections were calculated by the BEB model [19] for comparison (the $x$ axis of these data was multiplied by the ratio of proton mass $m_{\mathrm{p}}$ to the electron-projectile mass $m_{\text {proj }}$ such that data for particles of the same velocity are compared).

by electron-capture processes. The authors normalized their data to those of electron-impact cross sections. Rudd et al. [32] measured the number of electrons produced by a proton traversing a gas volume, thereby not taking into account per se electron-capture processes. The same method was used by De Heer et al. [30], while Crooks et al. [29] measured the double differential ionization cross sections and obtained the total ionization cross section by integrating over electron energy and scattering angle.

In the higher energy range (above about $1 \mathrm{MeV}$ ), the proton cross sections measured by Rudd et al. and Knudsen et al. also agree well with those of electrons of equal velocity. This is consistent with the theoretical expectation based on the first Born approximation, where plane waves are used to describe the initial and final states of a bare projectile. According to Bethe [11], this approximation is applicable if the projectile potential constitutes a small perturbation, as is the case for projectiles of low charge and high velocity (much higher than the velocity of the target electrons). Using the first Born approximation, Bethe showed that the ionization cross section of a bare nucleus moving with velocity $v$ is proportional to the number of electrons $Z_{\text {targ }}$ of the target molecule and to the square of the projectile charge $Z_{\text {proj }}$, such that

$$
\sigma_{\text {ion }} \propto \frac{Z_{\text {targ }} Z_{\text {proj }}^{2}}{\beta^{2}} \ln \left(\beta^{2}\right),
$$

where $\beta=v / c$. This relation is independent of the particle type and was previously shown to hold for electrons with energies above $300 \mathrm{eV}$ and for protons with energies greater than $550 \mathrm{keV}$ [35] (see also Fig. 3).

For proton energies below about $1 \mathrm{MeV}$, the ionization cross section is higher than that for electrons of the same velocity. This is due to exchange interactions between incoming and bound electrons and the smaller energy range of secondary electrons in the case of electron impact [28]. For decreasing 


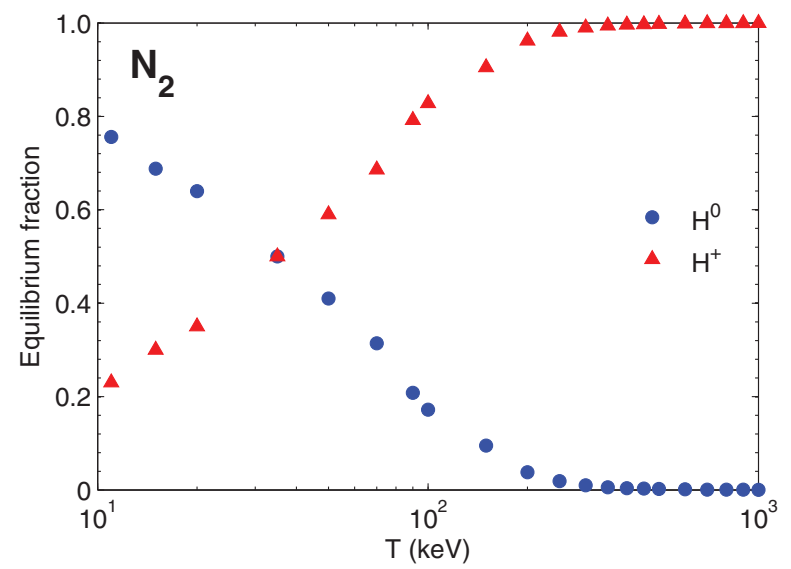

FIG. 4. (Color online) Equilibrium fractions of hydrogen particles $\mathrm{H}^{0}$ and $\mathrm{H}^{+}$traveling in nitrogen (reference in Table II).

proton energy, charge-transfer processes become increasingly important [33] (Fig. 4). In the energy range of interest in this work, however, the probability of an electron-capture process to produce a hydrogen atom is less than $20 \%$. Furthermore, the ionization cross section for neutral hydrogen atoms $\mathrm{H}^{0}$, measured by Puckett et al. [15], is about a factor of two lower than the proton cross section (see Fig. 3). For these reasons, charge-transfer processes for protons were not modeled in PTRA and protons were assumed to keep their charge state.

Figure 3 also shows cross section values obtained using two semiempirical models, which were developed and parametrized by Rudd et al. [28] and by Green and McNeal [34] (see Appendices C and D, respectively). In the following, these models are referred to as the Rudd model and the Green model. For proton energies below $2 \mathrm{MeV}$, both models reproduce the experimental data of all groups within the experimental uncertainties. At higher energies, the predictions by the Rudd model are in good agreement with the electron data, where the measured electron cross sections are well described by the BEB model within 10\% (see Sec. IV A). However, the discrepancy between the Green model and the BEB model increases with increasing energy, leading to about $30 \%$ higher values at $10 \mathrm{MeV}$ for the Green model. This deviation might be due to the limited energy range of the experimental data used by Green and McNeal to fit their model. From their publication it seems that measured data had only been available up to proton energies of about $1.5 \mathrm{MeV}$.

The Rudd model [28] was implemented in PTRA to parametrize ionization cross sections for protons in nitrogen, owing to the better agreement with the electron data at higher energies, which is in accordance with theoretical expectations based on the Bethe theory.

\section{Protons in propane and methane}

While ionization cross sections of propane for electron interactions have been determined by many groups (as discussed in Sec. IV B), no experimental data for light ions were found in the literature during this review. These cross section data for both protons and alpha particles, which are required for particle-track simulations, were obtained from those of methane (largely available) by using a scaling procedure.
TABLE III. Proton-impact ionization cross section data of methane and a semiempirical model for interpolation of the data. Data in $\mathrm{MeV}$.

\begin{tabular}{|c|c|c|}
\hline & Experimental & \\
\hline Ben-Itzhak et al. [36] & & $1-12.0$ \\
\hline Knudsen et al. [31] & & $0.5-6.0$ \\
\hline Luna et al. [37] & & $0.5-3.5$ \\
\hline Lynch et al. [38] & & $0.25,1$, and 2 \\
\hline Rudd et al. [32] & & $0.005-5.0$ \\
\hline \multicolumn{3}{|c|}{ Model } \\
\hline $\begin{array}{l}\text { Rudd model [28] } \\
\text { (see Appendix C) }\end{array}$ & & \\
\hline
\end{tabular}

Ionization cross sections of methane for protons were measured in the past by several groups [a selection is referenced in Table III and shown in Fig. 5(a)]. A comprehensive survey and evaluation of literature data has been published previously
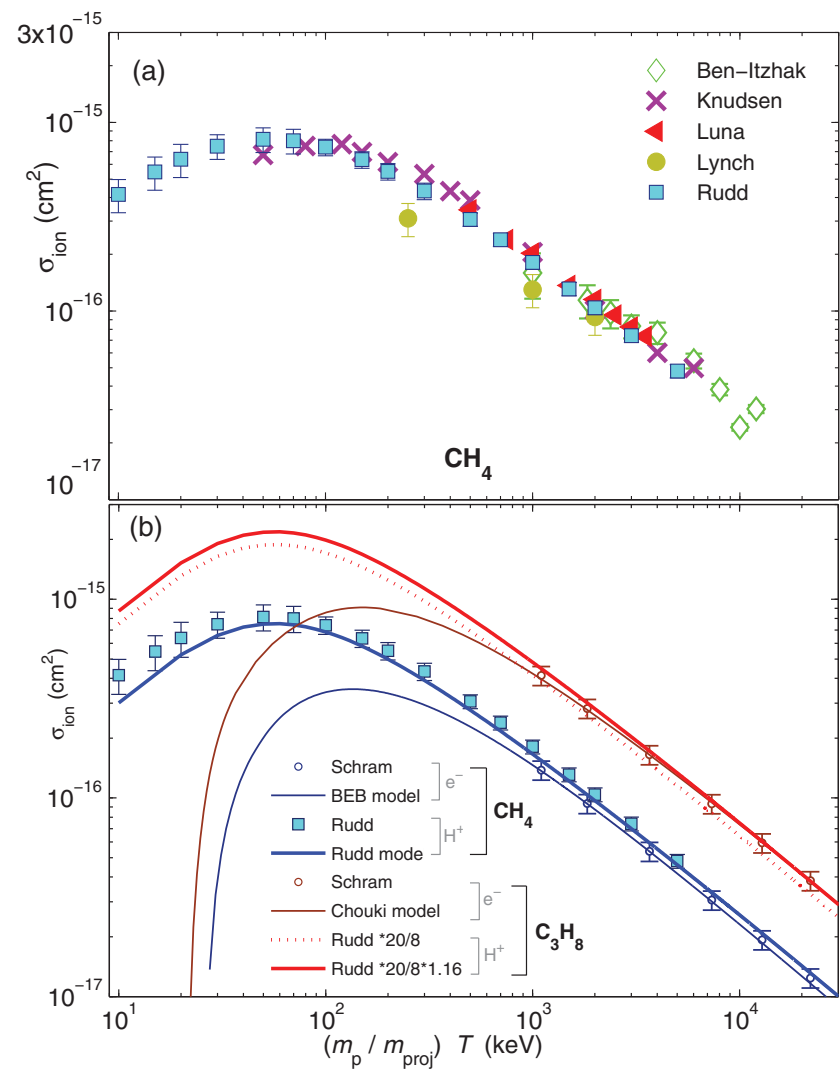

FIG. 5. (Color online) (a) Proton-impact-ionization cross sections $\sigma_{\text {ion }}$ of methane measured by the authors given in Table III. For the data of Luna et al., error bars are within the symbols. (b) Scaling of proton ionization cross sections of methane to propane. Symbols, experimental data; lines, results from model functions. For methane, a measured data set is shown as an example along with results from the Rudd model (references given in Table III). The scaled Rudd model for propane is also given. For comparison, electron-impact-ionization cross sections of Schram et al. and obtained by the BEB model and Chouki model are given for methane and propane, respectively (see Table I). Electron data were multiplied by the ratio of proton mass $m_{p}$ to electron-projectile mass $m_{\text {proj }}$ to achieve that both particle types are of the same velocity. 
[28]. Rudd et al. [32] measured the yield of secondary electrons produced by ionization of methane molecules by single traversing protons. Luna et al. [37] determined absolute cross sections for the production of charged methane fragments $\mathrm{CH}_{n}{ }^{+}(n=0-4)$ after an impact of protons by time-of-flight spectrometry. The sum of these cross section data is the total ionization cross section shown in Fig. 5(a). These data are in agreement with those of Rudd et al., within the experimental uncertainty of about 10\%. Ben-Itzhak et al. [36] used a similar method as Luna et al. to determine the relative yield of multiply charged methane fragments. The total ionization cross section data were also obtained by summing the cross sections for the production of methane fragments. Below about $3 \mathrm{MeV}$, the cross sections determined by Ben-Itzhak et al. [36] agree closely with the data of Luna et al. [37] and Rudd et al. [32], but when compared to the data of Rudd et al. at higher energies, they are nearly $20 \%$ larger.

The same kind of measurement was applied by Knudsen et al. [31] to determine the single-ionization cross section. The authors normalized their data to those of electron-impact cross sections in order to obtain absolute cross section values. Their results generally agree with those of Rudd et al. and Luna et al. within the experimental uncertainties across the entire energy range. As for nitrogen (see Fig. 3), the lowest two data points cause a shift of the maximum cross section to higher energies $(110 \mathrm{keV})$ when compared to the data of Rudd et al., who observed a maximum at about $70 \mathrm{keV}$. In the measurements of Knudsen et al., only single ionizations were taken into account, whereas the other authors included also multiple ionizations. Nevertheless, these data can be compared because the double-ionization cross section for methane was found to be two orders of magnitude smaller than the single-ionization cross section and therefore has a negligible contribution to the total ionization cross section [36]. Lynch et al. [38] determined the absolute ionization cross section for protons by an integration of the measured double differential ionization cross section as a function of secondary electron energy and scattering angle. Their data are lower than those of the other authors, deviating from those of Rudd et al. by as much as $40 \%$ at $250 \mathrm{keV}$.

Ionization cross section data of propane, missing in the literature, were implemented in the simulation code by multiplying the methane data by the ratio of the number of valence electrons in both molecules (i.e., 8 for methane and 20 for propane). Both methane and propane belong to the group of alkanes and thus have a similar bond structure. It can therefore be assumed that the relative energy dependence of the cross sections is similar. Scaling by the number of valence electrons of the target molecules is a reasonable approximation for particles of high velocities (above $1 \mathrm{MeV} / \mathrm{u}$ ), where the Born approximation is valid [38,39]. In fact, the probability of ionization of the inner shells by ion impact is much lower than that of the valence shells, and it can thus be assumed that only the valence electrons participate in the interaction. This can be seen in Fig. 5(b), where the ratio of the electron cross sections for propane to methane is about 2.7 at an energy of $550 \mathrm{keV}$, increasing to about a factor of 3 in the $\mathrm{MeV}$ range.

In order to obtain a suitable model for proton cross sections of propane for use in the simulations, the Rudd model for methane [28] was first scaled using the factor 2.5 (i.e., the ratio of the number of valence-shell electrons $Z_{\text {propane }} / Z_{\text {methane }}$ ) and then multiplied by an additional factor of 1.16 to match the electron data for propane at energies greater than about $550 \mathrm{keV}$ [Fig. 5(b)]. Thus, the ionization cross section data of propane used in the simulations for protons were determined by means of the Rudd model, which was parameterized for methane and multiplied by a total factor of 2.9.

\section{E. Helium particles in nitrogen}

\section{Ionization cross sections}

The available data for ionization cross sections of nitrogen for helium particles in the energy range of interest in this work are shown in Fig. 6(a) and references with respective energy ranges are listed in Table IV. In those experiments, a transverse electric field was used to collect electrons as well as positively charged ions produced by the projectile as it traversed the gas target, providing absolute cross sections for the production of electrons $\left(\sigma_{-}\right)$and slow positive ions $\left(\sigma_{+}\right)$, respectively. For $\mathrm{He}^{+}$and $\mathrm{He}^{0}$, electron-loss processes would contribute to the measured number of electrons, thereby yielding a cross section $\sigma_{-}$, which is larger than the ionization cross section. Similarly,

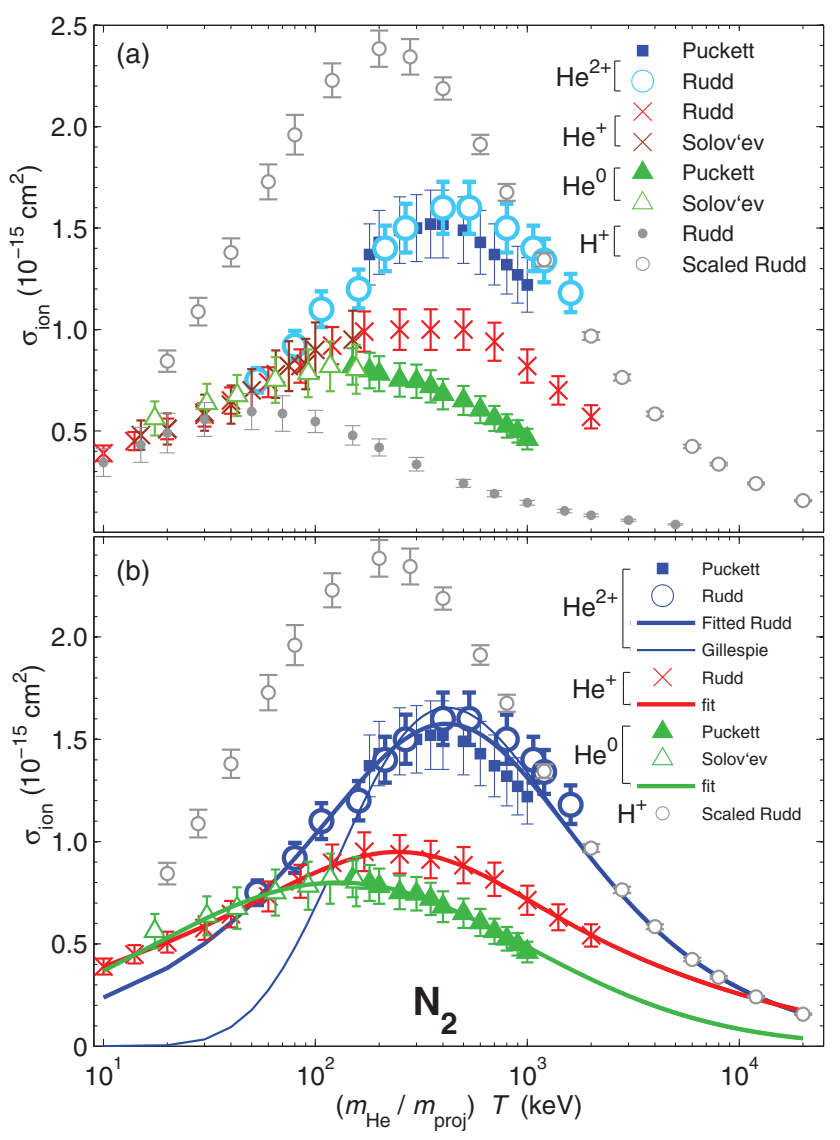

FIG. 6. (Color online) (a) Measured ionization cross sections $\sigma_{\text {ion }}$ of nitrogen for helium particles of different charge states (references given in Table IV). Proton data of Rudd et al. [32] are shown for comparison. To compare particles of the same velocity, the abscissa was also multiplied by the ratio of the masses of helium particles $\left(m_{\mathrm{He}}\right)$ and protons $\left(m_{\mathrm{p}}\right)$ and the ordinate was scaled by $Z^{2}$. (b) Models for an interpolation of the experimental data of nitrogen shown in (a) (see Table IV for references). 
TABLE IV. Impact-ionization and charge-transfer cross section data as well as equilibrium fractions for helium projectiles in nitrogen. Also listed are model functions and correction terms, investigated for an interpolation of cross sections. Data in $\mathrm{keV}$.

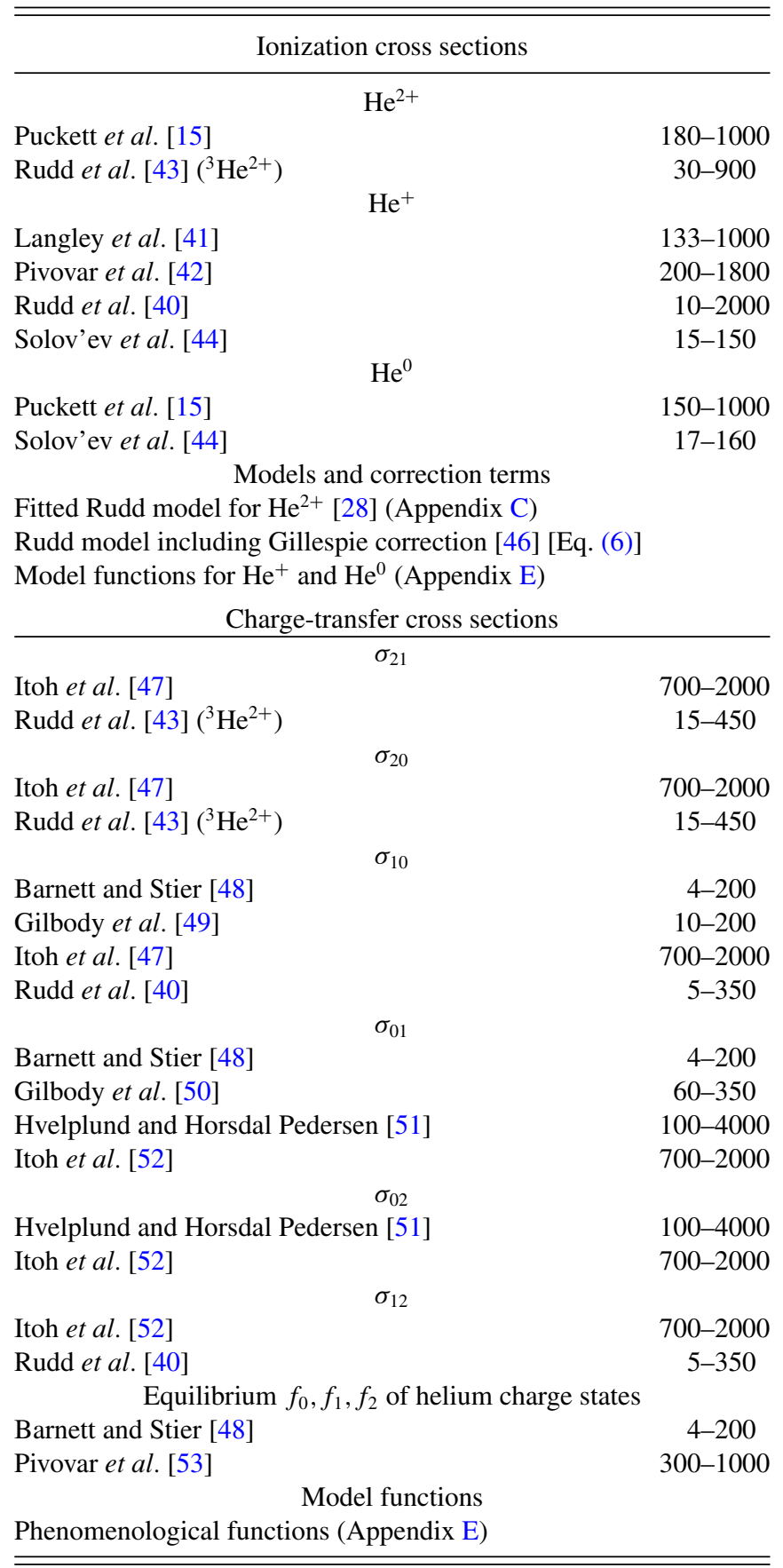

the ionization cross sections for $\mathrm{He}^{2+}$ and $\mathrm{He}^{+}$ions are lower than the respective cross sections for the production of positive charges. This is because positively charged target ions may be left behind, due to ionization of the target molecules by electron capture of the projectile. For $\mathrm{He}^{2+}$, the ionization cross section is equal to the cross section $\sigma_{-}$, as the electronloss probability is zero. Similarly, the ionization cross section for $\mathrm{He}^{0}$ equals $\sigma_{+}$, since the electron-capture cross section is negligible [15].
Since $\mathrm{He}^{+}$ions are able to capture or lose an electron, the cross section for single electron loss has to be subtracted from $\sigma_{-}$in order to obtain the desired ionization cross section. Therefore, the electron-loss cross section $\sigma_{12}$, determined by Rudd et al. [40], was subtracted from the $\sigma_{-}$cross sections, determined by the same authors [15,40-42]. The resulting ionization cross sections for $\mathrm{He}^{+}$ions were thereby reduced by a few percent at the lower energies and as much as $15 \%$ at about $1 \mathrm{MeV}$, where the electron-loss cross section has its maximum [Fig. 6(a)]. This reduction was still within the uncertainties associated with the experimental data of $\sigma_{-}$. It should be mentioned that ionization cross sections for $\mathrm{He}^{+}$ ions were determined by Langley et al. [41] and by Pivovar et al. [42] by assuming that the probability for electron-loss ionization (electron loss in combination with an ionization of the target molecule) is much higher than for electron loss only. Their ionization cross sections are, therefore, up to $30 \%$ smaller than $\sigma_{-}$(not shown).

The ionization cross sections for $\mathrm{He}^{2+}$ ions ${ }^{2}$ and ${ }^{3} \mathrm{He}^{2+}$, shown in Fig. 6(a), were measured by Puckett et al. [15] and by Rudd et al. [43], respectively. The cross sections by Rudd et al. were set to be equal to those of $\mathrm{He}^{2+}$ for the same velocity of both projectiles. For energies below about $800 \mathrm{keV}$, these data agree well with those of Puckett et al. (within the experimental uncertainties of $8 \%$ and $11 \%$ for the data of Rudd et al. and Puckett et al., respectively). At higher energies, the deviation reaches $17 \%$ at $1 \mathrm{MeV}$, which is still within the combined uncertainty values. The cross sections for $\mathrm{He}^{+}$ measured by Rudd et al. [40], Solov'ev et al. [44], and Pivovar et al. [42] (not shown to keep readability) agree well in the overlapping energy range. Those of Langley et al. [41] (not shown) are by as much as $25 \%$ larger with increasing deviation for decreasing energy. Only two sets of measured ionization cross section data for $\mathrm{He}^{0}$ were found in the literature, those by Puckett et al. [15] and by Solov'ev et al. [44]. Within the overlapping energy regions, the $\mathrm{He}^{+}$and $\mathrm{He}^{0}$ cross sections of the different groups are in excellent agreement and well within the experimental uncertainties, which are between $10 \%$ (Rudd et al.) and $15 \%$ (Solov'ev et al.).

Figure 6(a) also shows cross section data for $\mathrm{He}^{2+}$ ions obtained from those of protons $\mathrm{H}^{+}$with the same velocity, which were scaled by the square of alpha particle charge $Z^{2}$ according to Eq. (5). The figure shows that the ionization cross section for alpha particles is approaching the scaled cross section for protons at energies above $1 \mathrm{MeV}$, where the first Born approximation is valid. Below $1 \mathrm{MeV}$, the $\mathrm{He}^{2+}$ cross sections are lower than the scaled proton cross sections by as much as $45 \%$ at $100 \mathrm{keV}$. Furthermore, the maximum of the ionization cross section for the measured $\mathrm{He}^{2+}$ data occurs between 350 and $450 \mathrm{keV}$ (for the data of Puckett et al. and Rudd et al., respectively), while it is at about $70 \mathrm{keV}$ for protons, which corresponds to an energy of about $280 \mathrm{keV}$ for alpha particles of equal velocity.

The reduction of the cross section for low-energy alpha particles with respect to the scaled proton data is due to twocenter phenomena. The target-centered charge distribution of

\footnotetext{
${ }^{2}$ Note that the mass number of helium projectiles mentioned in this work is always 4 unless indicated otherwise.
} 
initially bound electrons adjusts adiabatically to the long-range Coulomb potential of the (slow) traversing projectile [45]. Due to the subsequent screening of the projectile potential by the attracted electron distribution, the emission probability of an electron decreases with decreasing projectile energy. Also, if the encounter of the projectile to the target is close, the projectile's potential reaches inside the orbit of the target electrons. This leads to an increased binding energy of the electrons and, subsequently, to a decrease in the ionization cross section. Two-center effects are not included in the first Born approximation and the ionization cross section is no longer proportional to the square of the nuclear charge $Z$ [as described by Eq. (5)]. Gillespie [46] proposed a simple model for an effective projectile charge $Z_{\text {eff }}$, which enables more realistic scaling between the proton and alpha particle cross sections,

$$
Z_{\text {eff }}^{2}=Z^{2} \exp \left(-\lambda Z \alpha^{2} / \beta^{2}\right),
$$

where $\alpha$ is the fine structure constant and $\beta=v_{\text {proj }} / c$.

In his work, Eq. (6) was fitted for an impact of different ions ( $Z=1-13$ ) on $\mathrm{H}_{2}$ and $\mathrm{He}$, where the value of $\lambda$ was 1 for $\mathrm{H}_{2}$ and 2 for He. Figure 6(b) shows that, down to $150 \mathrm{keV}$, a good agreement of the scaled proton cross section with the measured ionization cross section for alpha particles is obtained when the parameter $\lambda$ is adjusted to $7 / R$, where $R$ is $13.61 \mathrm{eV}$. Below this energy, the predicted values of the Gillespie model underestimate the experimental data, for example, at $100 \mathrm{keV}$ it is $35 \%$ lower.

As an alternative to this model for effective-charge correction, the Rudd model [28] was fitted to the $\mathrm{He}^{2+}$ ionization cross section, adjusting only the parameter $C_{\text {Rudd }}$ (see Table VII and Appendix C). As can be seen in Fig. 6(b), the resulting fit curve (referred to as fitted Rudd model in the following) is in good agreement with the measured data. Above $1 \mathrm{MeV}$, the fitted Rudd model agrees well with the original Rudd model (scaled by the square of alpha-particle charge). Ionization cross sections, calculated using the effective charge obtained according to the Gillespie model, are only as much as 5\% larger than the predictions of the fitted Rudd model in the region of the maximum (i.e., between about $400 \mathrm{keV}$ and $2 \mathrm{MeV}$ ) and $35 \%$ lower at $100 \mathrm{keV}$. In summary, the fitted Rudd model was implemented in PTRA due to its good agreement with experimental cross sections for alpha particle across a wide energy range.

The ionization cross sections for $\mathrm{He}^{0}$ and $\mathrm{He}^{+}$are not well described by the combination of the original Rudd model [28] and the effective charge obtained from the Gillespie model. Therefore, to facilitate the implementation of the ionization cross sections for $\mathrm{He}^{0}$ and $\mathrm{He}^{+}$in the code, Gaussian functions were found to provide the best fit to the experimental data [see Eq. (E1), as well as Fig. 6(b)]. The large deviation between the $\mathrm{He}^{+}$or $\mathrm{He}^{0}$ and the $\mathrm{He}^{2+}$ cross sections [refer to Fig. 6(a)] is due to the electron(s) in the bound projectile state screening the nuclear charge [14]. This screening effect results in an effective projectile charge, which decreases with increasing adiabatic radius (a measure for the distance between the projectile and the target electron) [54]. The adiabatic radius is a function of projectile velocity as well as of kinetic energy and binding energy of the electron that is ejected in the ionization process. In the case of $\mathrm{He}^{+}$, the effective projectile charge varies between 1 for small momentum transfers (producing secondary electrons of low energies in glancing collisions) and 2 for large momentum transfers (producing secondary electrons of high energies in close collisions), which preferably occur for low and high energetic projectiles, respectively. This can be seen in Fig. 6(a) for energies below about $60 \mathrm{keV}$, where the ionization cross sections for singly charged helium ions and protons are similar. Above this energy, the $\mathrm{He}^{+}$cross section data increase more rapidly, forming a broad maximum at about $200 \mathrm{keV}$, where the $\mathrm{He}^{+}$and the $\mathrm{He}^{2+}$ cross sections appear to converge at an energy of about $10 \mathrm{MeV}$, as suggested in [14]. This high-energy behavior was taken into account in the fitting procedure.

\section{Charge-transfer cross sections}

Charge-transfer cross sections of nitrogen have been frequently measured in the past, as referenced in Table IV. The cross sections for electron capture $\sigma_{j i}$ (transferring the helium projectile from a charge state $j$ to a lower state $i$ ) and those for electron loss $\sigma_{i j}$ are shown in Figs. 7(a) and 7(b), respectively. Gilbody et al. [49,50] determined the charge-transfer cross sections $\sigma_{10}$, as well as $\sigma_{01}$, by means of a beam attenuation technique, where the double electron loss was assumed to be negligible. Hvelplund and Horsdal Pedersen [51] measured the electron-loss cross sections $\sigma_{01}$ and $\sigma_{02}$ by means of the initial growth method, in which the fractions of charge states (produced by an incident helium particle of specific charge)

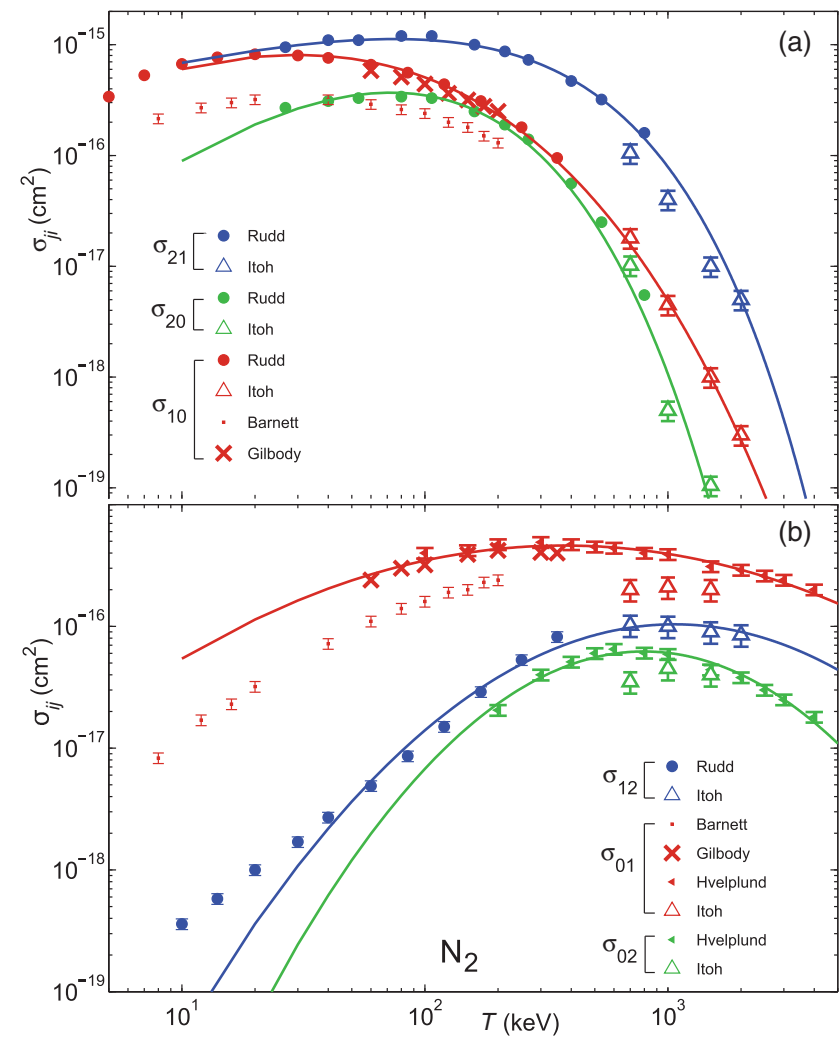

FIG. 7. (Color online) Cross section data of nitrogen for (a) electron-capture $\sigma_{j i}$ and (b) electron-loss $\sigma_{i j}$ processes of helium projectiles, measured by the authors referenced in Table IV (symbols). For some data sets error bars are within the symbols. The lines show the data obtained by the model functions given in Appendix E. 
were measured for different densities of the target gas. Using the same method, Rudd et al. [40,43] determined the single and double electron-capture cross sections $\sigma_{21}$ and $\sigma_{20}$ for ${ }^{3} \mathrm{He}^{2+}$, as well as the cross sections for single electron capture and loss for $\mathrm{He}^{+}, \sigma_{10}$ and $\sigma_{01}$, respectively. The electron-capture cross sections for $\mathrm{He}^{2+}$ were obtained from those for ${ }^{3} \mathrm{He}^{2+}$ of the same velocity. The cross section data determined by these authors are in good agreement within the stated experimental uncertainties, which were about $10 \%$.

In an early publication, Barnett and Stier [48] measured the cross sections $\sigma_{10}$ and $\sigma_{01}$, using the initial growth method. Their results are approximately two times lower than those reported by other authors. It is, however, interesting that these data also appear in a publication by Allison [33] but in comparative studies published by Gilbody et al. $[49,50]$ and Hvelplund and Horsdal Pedersen [51] they are a factor of two larger. Considering that the data obtained by Barnett and Stier for other molecules $\left(\mathrm{H}_{2}, \mathrm{He}, \mathrm{Ne}, \mathrm{Ar}\right)$ agree well with (and in some cases are even larger than) those determined by the other two groups, it seems possible that a correction factor of two may have been applied to their data for nitrogen. In this case, their data are up to $15 \%$ and $30 \%$ lower than the data of Rudd et al. [40] for $\sigma_{01}$ and $\sigma_{10}$, respectively. Itoh et al. [47,52] also used the initial growth method to measure single and double electron-capture as well as electron-loss cross sections. Some of their data (i.e., $\sigma_{21}, \sigma_{01}$, and $\sigma_{02}$ ) are generally a factor of two lower than those of other authors and exhibit a different qualitative behavior, particularly for $\sigma_{01}$ and $\sigma_{02}$. For $\sigma_{12}, \sigma_{10}$, and $\sigma_{20}$ no data were available for comparison during the compilation of this work, at least in the energy range of interest.

Experimental data were fitted by exponential and polynomial functions [given in Eqs. (E2) and (E3)], excluding the data from Barnett and Stier and the two electron-loss cross section data of Itoh et al. mentioned above. The electron-capture cross sections $\sigma_{21}$ of Itoh et al. were given a lower weight of 0.5 , as these were a factor of two lower than other data. The fitted curves are the solid lines shown in Fig. 7.

In order to test the consistency of these fitted cross sections, the equilibrium fractions $f_{0}, f_{1}$, and $f_{2}$ (which correspond to the three helium charge states $\mathrm{He}^{0}, \mathrm{He}^{+}$, and $\mathrm{He}^{2+}$, respectively), as measured by Barnett and Stier [48] and Pivovar et al. [53], were compared to the charge-transfer cross section data calculated using Eq. (3). This comparison, shown in Fig. 8, included three different approaches.

(v1) The measured equilibrium fractions were fitted by the functions summarized in Eqs. (E4a)-(E4c). These were used, together with the functions fitted to the electron-capture cross sections $\sigma_{10}, \sigma_{20}$, and $\sigma_{12}$, to determine the electron-loss cross sections $\sigma_{01}, \sigma_{02}$, and $\sigma_{21}$ by means of Eq. (3).

(v2) The functions fitted to the cross sections $\sigma_{01}, \sigma_{10}, \sigma_{12}$, and $\sigma_{21}$ were used to calculate the equilibrium fractions of the helium charge states by means of Eq. (3).

(v3) The same approach as (v2), except that the cross sections $\sigma_{01}, \sigma_{10}, \sigma_{02}$, and $\sigma_{20}$ were used.

In approaches (v2) and (v3) the charge-transfer cross section data for $\mathrm{He}^{+}$were used, since the agreement of the fitted models with the experimental data appear to be most reliable. In all three approaches, the fraction $f_{1}$ was chosen to be determined by subtracting $f_{0}$ and $f_{2}$ from unity [see Eq. (4)].

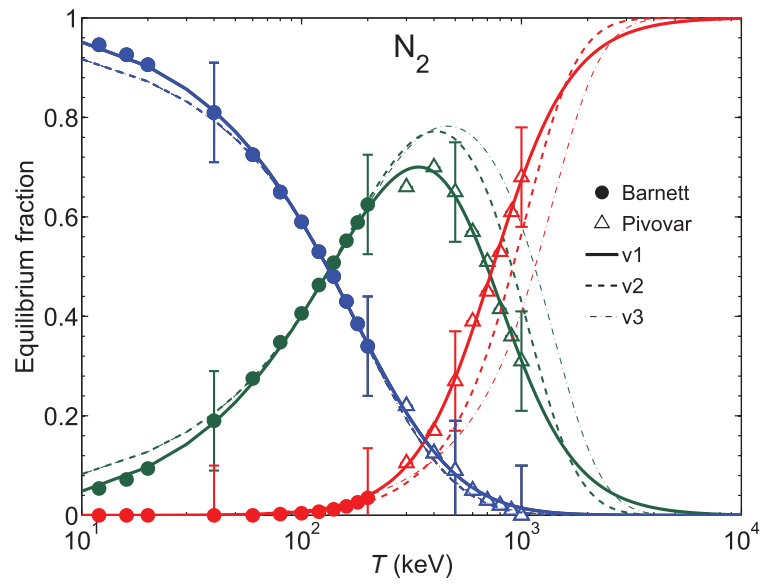

FIG. 8. (Color online) Equilibrium fractions $f_{0}$ (blue), $f_{1}$ (green), and $f_{2}$ (red) of helium particles traveling in nitrogen. Measured fractions (symbols) and fractions obtained by the model functions (solid lines) are shown (for references, see Table IV). Equilibrium fractions were also calculated from charge-transfer cross section data [(v2) and (v3)], as described in Sec. IV E2.

Up to an energy of $200 \mathrm{keV}$, the equilibrium fractions determined from the fitted models [approach (v1)] agree well with the data measured by Barnett and Stier, deviating by less than 5\% (Fig. 7). Above this energy, the equilibrium fractions of $\mathrm{He}^{+}$and $\mathrm{He}^{2+}$ measured by Pivovar et al. differ by up to $15 \%$ from those determined by approach (v2). An even larger deviation of up to $25 \%$ occurs for energies between $200 \mathrm{keV}$ and $3 \mathrm{MeV}$ using approach (v3), revealing some inconsistency of the data in the literature. For helium particles of energies above $2 \mathrm{MeV}$, the probability for electron capture can be neglected in all three approaches as the fraction of $\mathrm{He}^{2+}$ ions with these energies is greater than $95 \%$. Due to their large deviations, (v1) and (v3) are used in Secs. IV G and $\mathrm{V}$ to investigate the sensitivity of simulation data on the different equilibrium fractions. Recommendations for the implementation of the cross sections are then given in Sec. VI.

\section{F. Helium particles in propane and methane}

\section{Ionization cross sections}

There are no published experimental ionization cross section data of propane for helium projectiles. Rudd et al. $[40,43]$, however, measured $\sigma_{-}$of methane for $\mathrm{He}^{2+}$ and $\mathrm{He}^{+}$ ions (see Table V). The ionization cross sections of methane can be scaled to obtain those of propane according to Bethe's theory (refer to Sec. IV C).

The Rudd model [28] was used to fit the $\mathrm{He}^{2+}$ ionization cross section of methane by varying the parameter $C_{\text {Rudd }}$ (see Appendix C and compare to Sec. IV E1). As can be seen in Fig. 9, the data calculated using the fitted Rudd model are in good agreement with the measured data as they are within the experimental uncertainty of about $8 \%$. Also plotted are the cross section data obtained by the original Rudd model with a correction term for the effective charge, $Z_{\text {eff }}$, according to the Gillespie model using the parameter $\lambda=10$ [see Eq. (6)]. The predicted values of this model are within the experimental uncertainty for energies above $110 \mathrm{keV}$ but steeply decrease with decreasing energy, such that it already deviates by $15 \%$ 
TABLE V. Impact-ionization and charge-transfer cross section data as well as equilibrium fractions for helium projectiles in methane and propane. Also listed are models and correction terms, investigated for an interpolation of cross sections. Data in $\mathrm{keV}$.

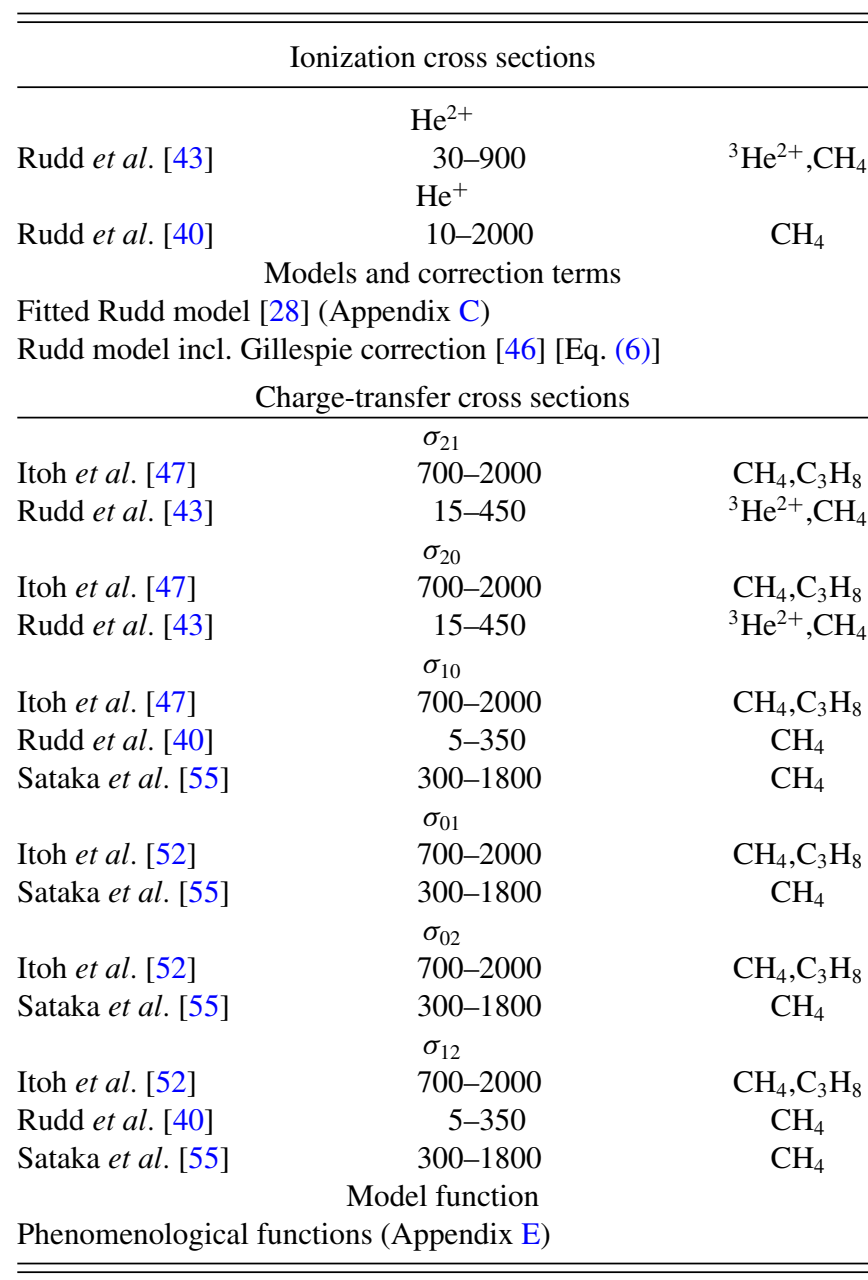

at $100 \mathrm{keV}$ from the measured data. The maximum of the $\mathrm{He}^{2+}$ cross sections predicted by these models occurs at about $350 \mathrm{keV}$, which is similar to the energy of the maximum in the nitrogen cross section data.

The $\mathrm{He}^{+}$ionization cross sections of methane were determined from the $\sigma_{-}$data by subtracting $\sigma_{12}$, which was also measured by Rudd et al. [40] (see also Sec. IV E1). The $\mathrm{He}^{+}$ionization cross section data were then fitted using a superposition of two Gaussian functions, given by Eq. (E1). Ionization cross sections of propane for alpha particles and $\mathrm{He}^{+}$ions were then obtained by scaling the corresponding cross sections of methane by a factor of 2.9 (see Sec. IV D, where this factor was previously used when scaling the proton ionization cross section data of methane to those of propane).

For $\mathrm{He}^{0}$ atoms, ionization cross section data of both methane and propane could not be found in the literature. Figure 9, however, reveals that the $\mathrm{He}^{+}$ionization cross sections of nitrogen and methane have a similar energy dependence and deviate as much as $28 \%$ in the energy region between $170 \mathrm{keV}$ and $1.4 \mathrm{MeV}$ (mostly within the experimental uncertainties of $10 \%$ ). Due to this similarity and the lack of data, the ionization cross sections of methane for $\mathrm{He}^{0}$ were assumed to be equal to those of nitrogen. The

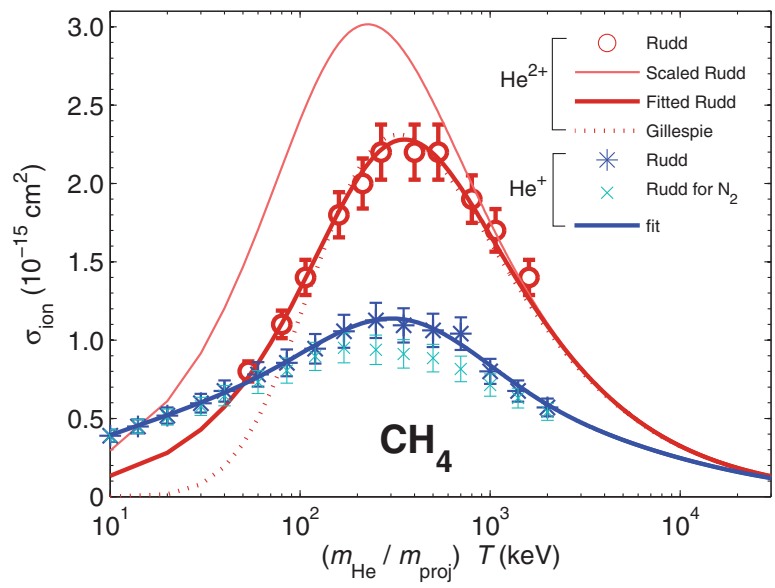

FIG. 9. (Color online) Ionization cross sections of methane for helium projectiles (symbols, experimental data; lines, model functions). Shown are the data referenced in Table $\mathrm{V}$ as well as the Rudd model [28] for protons with the same velocity as the helium particles (their abscissa was multiplied by the ratio of the masses of helium particles $m_{\mathrm{He}}$ to protons $m_{\text {proj }}$ and the ordinate was scaled by $Z^{2}$ ). The measured ionization cross sections of nitrogen for $\mathrm{He}^{+}[40]$ is shown for comparison.

ionization cross sections of propane for $\mathrm{He}^{0}$ were then obtained by multiplying the cross sections of nitrogen by the factor of 2.9 .

\section{Charge-transfer cross sections}

Measured electron-capture and electron-loss cross sections for methane and propane are also listed in Table $\mathrm{V}$ and shown in Fig. 10, together with the different models used previously to fit the nitrogen cross sections (see Sec. IV E and Appendix E). The single electron-capture and electron-loss cross sections of methane for $\mathrm{He}^{+}$ions, $\sigma_{10}$ and $\sigma_{12}$, were measured for $\mathrm{He}^{+}$ions by Rudd et al. in the energy range between 5 and $350 \mathrm{keV}$ [40]. These data are about $20 \%$ lower than those measured by Sataka et al. [55], judging by the small overlap of the energy ranges. Electron-capture cross sections of methane for ${ }^{3} \mathrm{He}^{2+}, \sigma_{21}$ and $\sigma_{20}$, were also measured by Rudd et al. [43]. The ${ }^{3} \mathrm{He}^{2+}$ cross sections were used to determine the electron-capture cross sections for ${ }^{4} \mathrm{He}^{2+}$ of the same velocity. In the energy range between $700 \mathrm{keV}$ and 2 $\mathrm{MeV}$, the electron-capture cross sections are complemented by the data of Itoh et al. [47]. The electron-loss cross sections $\sigma_{01}$ and $\sigma_{02}$ of methane were measured by Sataka et al. [55], but are up to a factor of two larger than those determined by Itoh et al. [52]. In fact, only Itoh et al. measured all six charge-transfer cross sections for methane.

It is worth noting that the fits of the charge-transfer cross section data of nitrogen for helium particles (Fig. 10) generally also reproduce the measured data for methane with satisfactory agreement. Exception are the electron-loss cross sections $\sigma_{02}$ of methane measured by Sataka et al. and all of the methane data of Itoh et al., which are a factor of two lower than those calculated with the nitrogen models. The agreement of charge-transfer cross sections of nitrogen with those of methane is, however, plausible because they strongly depend on the velocity distribution of valence electrons in 


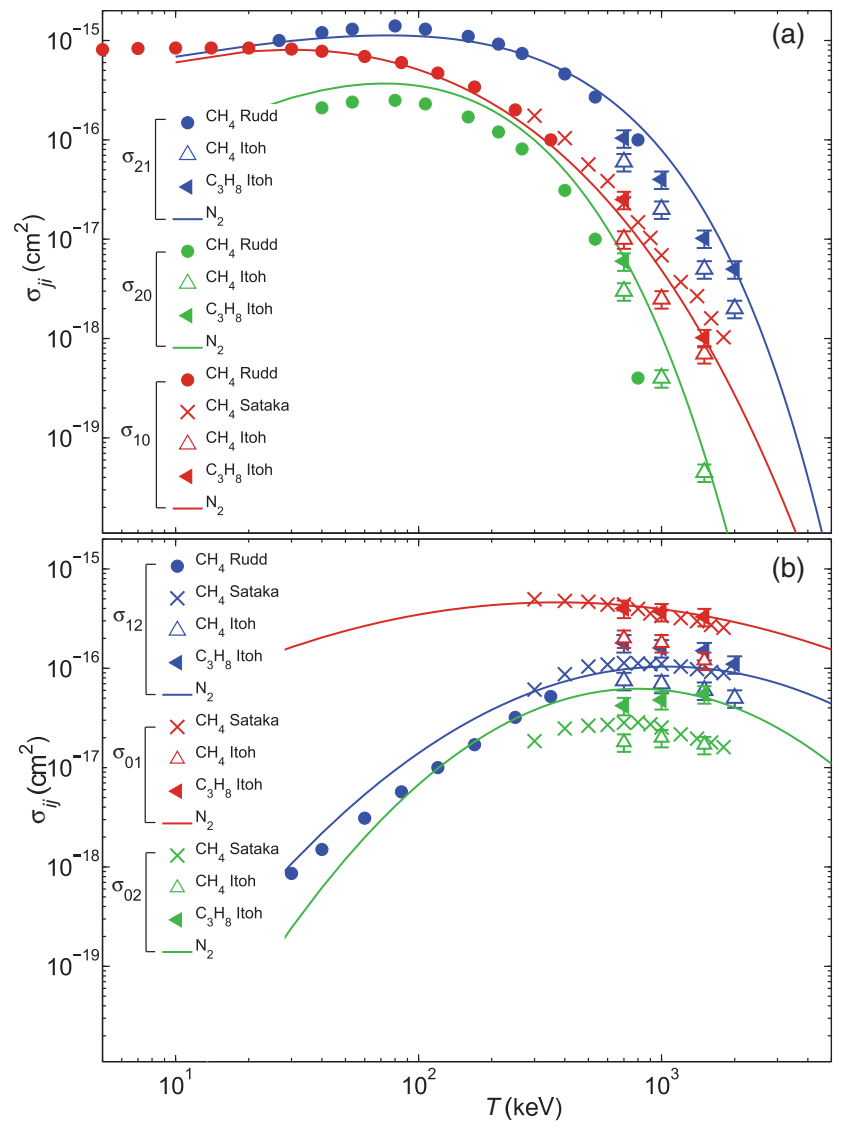

FIG. 10. (Color online) Cross section data for (a) electron capture $\sigma_{j i}$ and (b) electron loss $\sigma_{i j}$ of methane $\mathrm{CH}_{4}$ and propane $\mathrm{C}_{3} \mathrm{H}_{8}$ for helium projectiles, measured by the authors referenced in Table $\mathrm{V}$ (symbols). For some data sets error bars are within the symbols. The lines show the model functions fitted to the nitrogen data given in Appendix E.

the target with respect to the projectile velocity. For example, electron-capture processes for helium particles are maximum when the velocity of the projectile corresponds to the velocity of an outer valence electron in the medium. The energy-loss processes, on the other hand, can be interpreted as a projectile ionization by the target potential, which is largely determined by the binding energies of the valence electrons. In fact, the binding energies of the ten or eight valence electrons in nitrogen and methane, respectively, are quite similar [20], so that the same charge-transfer cross sections can be expected. On the other hand, propane has 20 valence electrons with binding energies of the six outermost well below those of nitrogen or methane [20].

For propane, the only available data set for charge-transfer cross sections for helium particles was published by Itoh et al., who measured all six charge-transfer cross sections (see again Table V and Fig. 10). Their cross sections for propane are generally about a factor of two larger than their methane data. However, their propane data are in agreement with measured methane cross sections of other authors, while their methane cross section data are generally a factor of two lower than those determined by other groups (see discussion in the previous subsections). The same tendency was observed for most of their nitrogen data in Sec. IV E2. These findings indicate that their data for propane may be about a factor of two too low. Itoh et al., however, determined their data in a narrow energy region between 0.7 and $2 \mathrm{MeV}$ with only one to four data points, so that, in our point of view, these can only be interpreted as being supportive to the relative energy dependence of the methane cross section data of the other authors. Therefore, the nitrogen model functions were also used to model the charge-transfer cross sections of propane. However, the influence of a factor of two larger charge-transfer cross sections for propane was quantified for the nanodosimetric parameters studied in the PTRA simulations (see Sec. V A).

\section{G. Effective ionization cross sections for helium particles}

Target molecules are generally ionized by the three helium charge states $\left(\mathrm{He}^{0}, \mathrm{He}^{+}\right.$, and $\left.\mathrm{He}^{2+}\right)$ in either direct impactionization processes or electron capture to a bound state of the projectile. The resulting effective ionization cross section $\sigma_{\text {eff }}$ for a given helium particle energy is then the sum of the cross sections for those processes, weighted by the equilibrium fractions of the helium charge states (see Sec. III), such that

$$
\sigma_{\text {eff }}=f_{0} \sigma_{\mathrm{He}^{0}}+f_{1}\left(\sigma_{\mathrm{He}^{+}}+\sigma_{10}\right)+f_{2}\left(\sigma_{\mathrm{He}^{2+}}+\sigma_{21}+\sigma_{20}\right) \text {. }
$$

The equilibrium fractions $f_{0}, f_{1}, f_{2}$ were taken from the functions fitted to the experimental data [see Eqs. (E4a)-(E4c) and Fig. 8]. For the calculation of $\sigma_{\text {eff }}$, the ionization cross sections of nitrogen and methane for alpha particles $\sigma_{\mathrm{He}^{2+}}$ were determined from the fitted Rudd model. For $\mathrm{He}^{+}$and $\mathrm{He}^{0}$, the ionization cross sections were obtained using Eq. (E1). The $\mathrm{He}^{0}$ ionization cross sections of nitrogen were also used for methane, owing to the similarity of the $\mathrm{He}^{+}$ionization cross section data of methane and nitrogen (Fig. 9) and the lack of respective literature data. The charge-transfer cross sections for nitrogen and methane were obtained from Eqs. (E2) and (E3). Effective ionization cross sections were then determined for both nitrogen and methane using Eq. (7). The reader should be reminded that the ionization cross sections of propane for $\mathrm{He}^{2+}, \mathrm{He}^{+}$, and $\mathrm{He}^{0}$ were calculated using those of methane, multiplied by a factor of 2.9 (see Sec. IV F).

Figure 11 shows a comparison of different effective ionization cross sections ( $\sigma_{\text {eff }}$ ) of nitrogen to the corresponding measured ionization cross sections for $\mathrm{He}^{2+}$ ions, $\sigma_{\mathrm{He}^{2+}}$ [43]. For energies of $2 \mathrm{MeV}$ and higher, the measured proton cross sections of Rudd et al. [32] are also plotted (scaled to the same velocity as an alpha particle and multiplied by the square of the alpha particle's charge). The effective ionization cross section of nitrogen has a maximum at about $700 \mathrm{keV}$ where it is about $30 \%$ lower than the ionization cross section of alpha particles. Beyond the maximum, the effective ionization cross sections approach the scaled ionization cross section for protons and coincide at $2 \mathrm{MeV}$. This is due to the low equilibrium fractions of $\mathrm{He}^{+}$and $\mathrm{He}^{0}$ particles at high energies. Below the energy of the maximum, the effective cross section decreases slightly with decreasing energy, coinciding again with the alpha particle ionization cross section at $100 \mathrm{keV}$.

As an alternative to Eq. (7), an empirical model was developed by Barkas [56,57] to estimate the effective charge of the projectile arising from higher-order interactions as well as charge-transfer processes. Similar to the model of Gillespie 


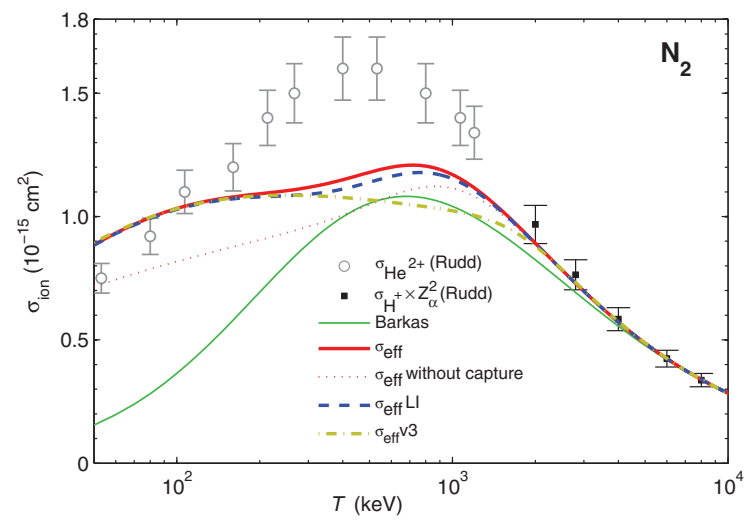

FIG. 11. (Color online) Effective ionization cross sections of nitrogen for helium particles $\left(\sigma_{\text {eff }}\right)$, determined using both the Barkas correction and Eq. (7). Shown are also effective cross sections, which would be obtained by omitting electron-capture processes, by considering loss ionization (LI) processes in the $\mathrm{He}^{+}$ionization cross section, and by using approach (v3) (see Sec. IV E). Further, ionization cross sections for alpha particles $\left(\sigma_{\mathrm{He}^{2+}}\right)$ and protons (abcissa scaled to give particles of same velocities and the ordinate was scaled by the square of the alpha particle charge, $\sigma_{\mathrm{H}^{+}} Z^{2}$ ) are compared.

[see Eq. (6)], it contains only a single fitting parameter. Barkas fitted the energy loss as a function of projectile velocity for different ions in silver halide emulsions,

$$
Z_{\text {eff }}^{2}=Z^{2}\left[1-\exp \left(-a \beta Z^{-2 / 3}\right)\right]^{2},
$$

where $\beta=v_{\text {proj }} / c, v_{\text {proj }}$, and $c$ are the velocity of the projectile and light, respectively. The adjustable parameter $a=125$ was found by Barkas and co-workers to be applicable for a wide range of projectiles, target gases, and solids [56].

The Barkas model is widely used in the literature to determine ionization cross sections for slow ions [58]. In this work, however, it was observed that the application of the Barkas correction is rather unsatisfactory. Multiplying the square of the effective charge with the energy-scaled proton cross section leads to a much lower effective ionization cross section than previously obtained: At about $2 \mathrm{MeV}$ it is already by about $10 \%$ lower, showing also a maximum at $700 \mathrm{keV}$, and decreases for lower energies to about $1 / 3$ of the alpha particle cross sections which coincide with the previously obtained effective cross section at $100 \mathrm{keV}$.

The divergence between ionization cross sections obtained using the Barkas model or $\sigma_{\text {eff }}$ is, in part, due to the contribution of electron-capture processes to the latter cross section. When the contribution of electron-capture processes was omitted from Eq. (7) (Fig. 11), a reduction of up to $27 \%$ (at $100 \mathrm{keV}$ ) in the effective ionization cross sections of nitrogen and up to $10 \%$ for propane (not shown) are observed. The influence of electron-capture processes on the effective ionization cross section of propane is less than that for nitrogen. This is because only the ionization cross sections of propane were scaled (using the methane cross sections), while for both methane and propane the same models for the electron-capture cross sections fitted to the nitrogen data were used. The applicability of the Barkas model to obtain nanodosimetric quantities from track structure simulations is discussed in Sec. V.

Furthermore, several authors suggested that electron-loss ionization (LI) contributes to the measured data for the impact of $\mathrm{He}^{+}$ions (see Sec. IVE1). To investigate a possible influence on the effective ionization cross sections, it was assumed in this work that $50 \%$ of the collected charges are due to single electron-loss processes and 50\% include an additional ionization of the target molecules. Based on this assumption, the ionization cross sections of $\mathrm{He}^{+}$were recalculated. Subsequently, the effective ionization cross sections obtained by assuming single electron loss only results in being reduced by up to $3.5 \%$ and $8 \%$ for nitrogen and propane, respectively, in the energy range between $100 \mathrm{keV}$ and $2 \mathrm{MeV}$.

Another important question is how the different equilibrium fractions shown in Sec. IV E2 influence the effective ionization cross section. For this purpose, the equilibrium fractions in nitrogen as well as the electron-capture cross section $\sigma_{21}$ used in Eq. (7) were calculated as described in Sec. IV E2 (v3) [i.e., the fits of the cross sections $\sigma_{01}, \sigma_{10}, \sigma_{02}$, and $\sigma_{20}$ were used to calculate the equilibrium fractions of the helium charge states by means of Eq. (3)]. Note that single electron loss only was assumed. This procedure led to a quite different energy dependence of the equilibrium fraction than obtained from measurements (v1), revealing some inconsistency of the charge-transfer data in the literature (see Fig. 8). As expected, the influence on the effective ionization cross section is significant with a reduction in the energy range between $200 \mathrm{keV}$ and $2 \mathrm{MeV}$ (by as much as $16 \%$ at $800 \mathrm{keV}$ ). The effect on simulation results of the deviation between the effective ionization cross sections obtained by approaches (v1) and (v3) is shown in the following section.

For propane, only one data set for charge-transfer cross sections was found in the literature, which may be a factor of two higher than those of methane (see Sec. IV F2). Including this assumption in Eq. (7), an increase of electron-capture cross sections by a factor of two would increase the effective ionization cross section for energies below $1 \mathrm{MeV}$. At energies below $100 \mathrm{keV}$, this amounts to $8 \%$ (Fig. 11). The effect of this factor on simulation results is shown in Sec. V.

\section{SUITABILITY OF CROSS SECTION DATA INTERPOLATIONS FOR PARTICLE-TRACK SIMULATIONS}

The suitability of the reviewed cross section data for calculating realistic particle-track parameters with the code PTRA was investigated by benchmarking simulated nanodosimetric quantities with experimental results obtained with the PTB/WIS nanodosimeter (see Sec. III). In particular, the mean ionization cluster size, $M_{1}$, and the total energy loss were calculated for protons and alpha particles of different energies, with the nanodosimeter being operated with either nitrogen or propane. Also, the influence of different ionization cross section models for alpha particles on those quantities was investigated in this work, with the purpose of assessing the data robustness and to give a rough estimate on associated model uncertainties. 
The following models for describing different ionization cross sections for helium particles were implemented in the PTRA code and tested for suitability.

(i) The Rudd model [28] was fitted to the average of measured ionization cross sections of alpha particles (see Sec. IVE and Appendix C). These data are referred to as fitted Rudd model in the following.

(ii) The proton ionization cross section was multiplied by the square of an effective charge, $Z_{\text {eff }}^{2}$, according to the Barkas model [56], using $a=125$ for both nitrogen and propane (see Sec. IV G).

(iii) Ionization cross sections for alpha particles were obtained by the fitted Rudd model and, additionally, all the charge-transfer processes and ionization cross sections of $\mathrm{He}^{0}$ and $\mathrm{He}^{+}$(see Appendix E) were taken into account during the interaction sampling.

(iv) Effective ionization cross sections $\sigma_{\text {eff }}$ were used as described in Sec. IV G.

In all simulations, it was assumed that both ionization and electron-capture processes (when applied) contribute to the ionization cluster size distributions, since the target molecule is ionized in both cases.

\section{A. Mean ionization cluster size $M_{1}$ \\ 1. $M_{1}$ for nitrogen}

Figure 12 shows the measured and calculated mean ionization cluster size, obtained for protons and alpha particles in nitrogen as a function of the projectile energy (see also Refs. $[59,60])$. For protons, measured and simulated results agree within the experimental uncertainties (up to 14\%) for energies above $300 \mathrm{keV}$. Below this energy, the simulated cluster sizes are up to $17 \%$ lower than the measured data. Even though the model function applied in PTRA for the ionization cross section data in this energy region agrees well with the respective experimental data (refer to Sec. IV C), the uncertainties of those data were between $10 \%$ and $25 \%$. Keeping this in mind, the benchmark test based on the $M_{1}$ values for protons in nitrogen was successful. The recently published comparison of simulated and measured distributions of ionization cluster sizes for protons demonstrated also a good agreement [60].

A similar behavior can be observed for alpha particles. The values of $M_{1}$, calculated with PTRA using the fitted Rudd model reproduce the measured data well within the experimental uncertainties (which are between 7\% and 22\%) for energies above $1 \mathrm{MeV}$. Below this energy, the calculations yield values up to $23 \%$ larger than the measured data. The ratio of measured to simulated data in Fig. 12(b) reveals that the relative discrepancy rises with decreasing projectile energy. The maximum calculated value of $M_{1}$ appears at about $400 \mathrm{keV}$, which is slightly lower than $500 \mathrm{keV}$ observed in the experiments. The deviations found in such an artificial simulation (transporting, in fact, only alpha particles) illustrate the importance of considering charge-transfer interactions in the simulations.

Another set of simulation results obtained for incident alpha particles using the Barkas model compares well with the measured data down to an energy of about $2 \mathrm{MeV}$. With decreasing energy, however, $M_{1}$ determined using the Barkas

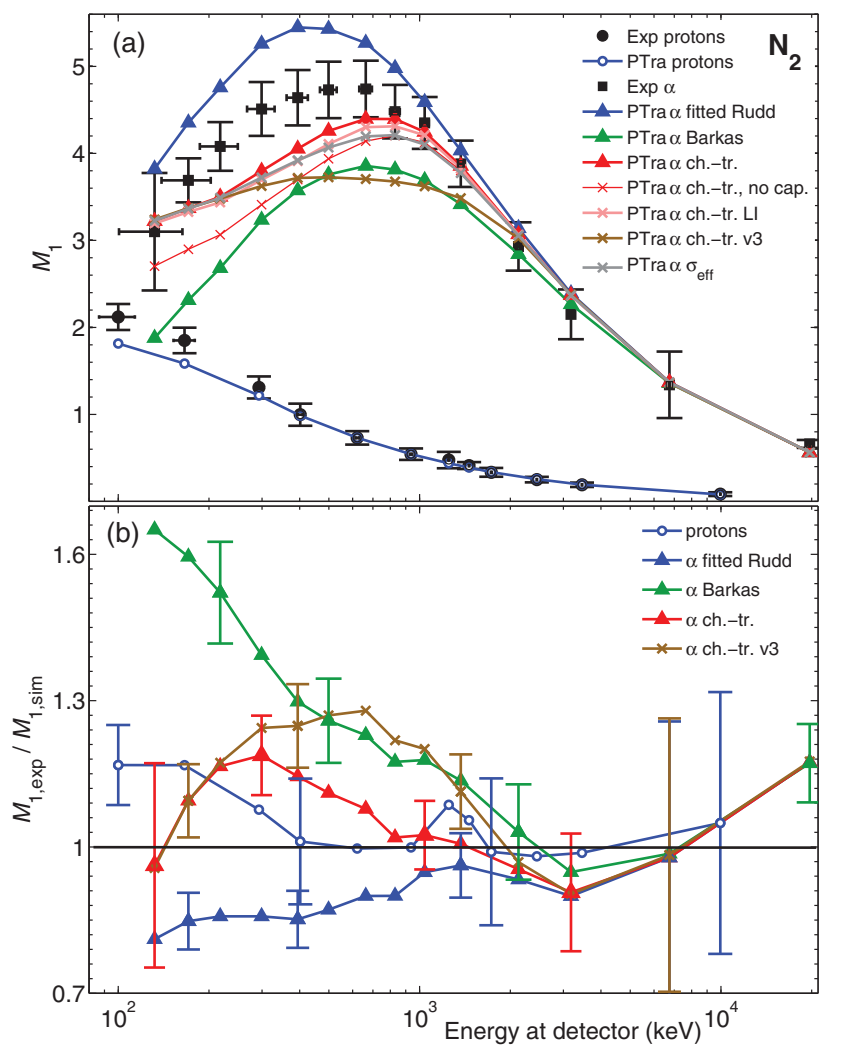

FIG. 12. (Color online) (a) Mean ionization cluster size $M_{1}$ of protons and alpha particles in nitrogen. The following cross section models for alpha particles were used: (i) fitted Rudd model (PTra $\alpha$ fitted Rudd); (ii) Barkas model for effective charge correction (PTra $\alpha$ Barkas); (iii) simulations including charge-transfer processes (PTra $\alpha$ ch.-tr.); (iv) as in (iii), but including only impact ionizations in $M_{1}$ (PTra $\alpha$ ch.-tr., no cap); (v) as in (iii), but taking LI into account in the $\mathrm{He}^{+}$ionization cross section (PTra $\alpha$ ch.-tr. LI); (vi) as in (iii), but using version (v3) to determine the equilibrium fractions of helium charge states (PTra $\alpha$ ch.-tr. v3); and (vii) using the effective ionization cross section $\sigma_{\text {eff }}$ (PTra $\alpha \sigma_{\text {eff }}$ ). (b) Ratio of the measured $M_{1, \exp }$ to simulated values $M_{1, \text { sim }}$ using (i) ( $\alpha$ fitted Rudd), (ii) ( $\alpha$ Barkas), (iii) ( $\alpha$ ch.-tr.), and (vi) ( $\alpha$ ch.-tr. v3). The statistical uncertainties of the simulations are contained within the symbols of the graphs.

model deviates dramatically from the experimental data. In fact, at the lowest helium particle energy (about $130 \mathrm{keV}$ ), the results from the Barkas model are a factor of 1.65 lower than the measured data. This finding supports the indication of an unsatisfactory correction of the effective charge of helium projectiles in the ionization cross sections of nitrogen (see Sec. IV G). On the other hand, a substantial deviation of results obtained including the Barkas model is due to the neglegted electron-capture processes when counting the number of ionizations. Considering those would increase the total $M_{1}$ value for helium particles of low energies. It is concluded that the Barkas model is not suitable for an application in track structure simulations for nanodosimetry.

Figure 12 also shows simulated $M_{1}$ values for alpha particles obtained with the inclusion of charge-transfer cross sections ("PTra $\alpha$ ch.-tr." in the figure). The experimental mean ionization cluster size is reproduced well for energies above $800 \mathrm{keV}$ but underestimated by up to $19 \%$ for energies 
between 150 and $800 \mathrm{keV}$. This deviation is, however, still within the overall experimental uncertainties of the cross section data (between $8 \%$ and 15\%) and equilibrium fractions (about 10\%). The maximum calculated $M_{1}$ is found at about $700 \mathrm{keV}$, which coincides with the maximum predicted by the Barkas model and is higher than the experimental maximum (at approximately $550 \mathrm{keV}$ ).

Substituting the charge-transfer and ionization cross sections for the different helium charge states by the effective ionization cross section $\sigma_{\text {eff }}$ (see Sec. IV G) gives similar results for $M_{1}$ in nitrogen. The largest deviation to $M_{1}$, obtained by accounting for charge-transfer processes, is $5 \%$ in the region of the maximum (labeled "PTra $\alpha \sigma_{\text {eff }}$ " in Fig. 12). The observed reduction of $M_{1}$ is due to the neglected transport of electrons produced in electron-loss processes of the helium projectiles in $\sigma_{\text {eff }}$. Electron-loss processes have broad maxima above $300 \mathrm{keV}$, which leads to the production of electrons with corresponding energies between 40 and $200 \mathrm{eV}$ as these electrons were assumed to have the same velocity as the projectile in the code. Electrons at such low energies have a small ionization mean free path (about $0.66 \mathrm{~mm}$ at $100 \mathrm{eV}$ for a pressure of $120 \mathrm{~Pa}$ in nitrogen). Therefore, they are likely to produce a significant number of ionizations within the sensitive volume (about $1 \mathrm{~mm}$ in diameter). As stated above, the contribution of electrons produced by electron-loss processes is, however, small due to the low charge-transfer cross section when compared with the ionization cross section. For example, if an $\mathrm{He}^{2+}$ ion experiences electron capture followed by electron loss, the respective cross sections $\sigma_{21}$ and $\sigma_{12}$ are both about an order of magnitude smaller than the corresponding ionization cross sections. Hence, the accuracy of the simulations is not significantly compromised when the cross section data set for ionization and charge-transfer processes are replaced by the effective ionization cross section (see Sec. IV G).

The contribution of electron-capture processes to the ionization of the target molecules was investigated to further evaluate the reason for the low $M_{1}$ values obtained using the Barkas model. For this purpose, the same cross section data set for ionization and charge transfer as above was used (i.e., "PTra $\alpha$ ch.-tr.") and only the number of impact ionizations per ion track was counted while ionizations arising from electron-capture interactions were not scored. The results of these simulations, also shown in Fig. 12, have a similar behavior as the cross section data shown previously in Sec. IV G: Compared to the results obtained by scoring electron-capture interactions (i.e., "PTra $\alpha$ ch.-tr."), the $M_{1}$ values are underestimated with decreasing alpha particle energies below $1 \mathrm{MeV}$ by up to $16 \%$. This deviation clearly shows that the contribution of ionizations produced in electron-capture interactions is significant for helium particles in this energy range. This contribution alone, however, does not entirely amount to the reduced $M_{1}$ values observed when using the Barkas model.

Furthermore, it has already been discussed in Sec. IV G that the impact-ionization cross sections of $\mathrm{He}^{+}$ions may be reduced by electron-loss ionization (LI) events. Hence, it was assumed that $50 \%$ of the literature cross sections for the production of positive ions or electrons are due to LI and $50 \%$ arise from single electron-loss processes. Nevertheless, as for the effective ionization cross section, the calculated reduction of $M_{1}$ values due to this assumption is not more than $3.5 \%$ and therefore not significant (labeled "PTra $\alpha$ ch.-tr. LI" in Fig. 12).

A significant reduction of the mean ionization cluster size in the energy region between $200 \mathrm{keV}$ and $2 \mathrm{MeV}$ by as much as $16 \%$ is observed when the equilibrium charge-state fractions are calculated as described in Sec. IV E2 (v3) ("PTra $\alpha$ ch.-tr. v3"). This is again consistent with the differences observed in the effective ionization cross section data (see Sec. IV G).

\section{2. $M_{1}$ for propane}

Measured and simulated mean ionization cluster sizes for protons and alpha particles in propane are shown in Fig. 13. For protons, calculated $M_{1}$ values favorably compare (within the experimental uncertainties of about 7\%) with the measured data across the entire energy range. Hilgers et al. $[10,60]$ demonstrated also a good agreement of simulated and measured distributions of ionization cluster sizes for protons.

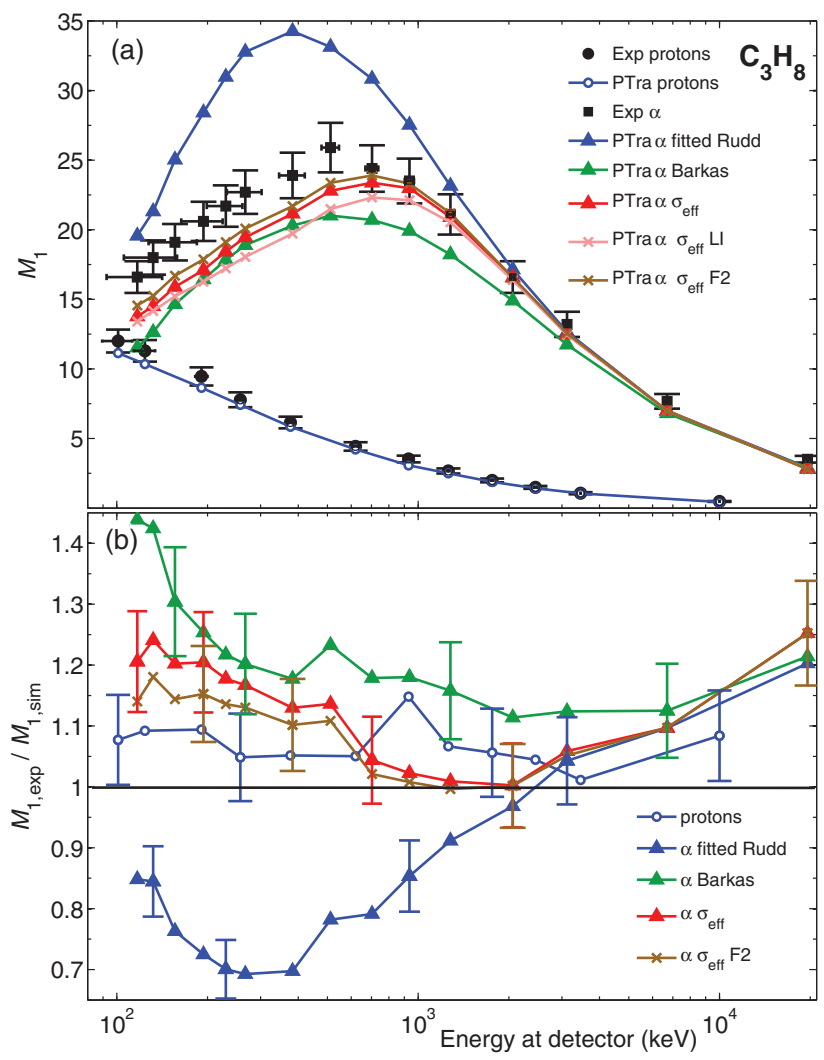

FIG. 13. (Color online) (a) Mean ionization cluster size $M_{1}$ of protons and alpha particles in propane. The following cross section models were used for alpha particles: (i) fitted Rudd model (PTra $\alpha$ fitted Rudd); (ii) Barkas model for effective charge correction (PTra $\alpha$ Barkas); (iii) effective ionization cross section $\sigma_{\text {eff }}$ (PTra $\alpha \sigma_{\text {eff }}$ ); (iv) $\sigma_{\text {eff }}$, taking $\mathrm{LI}$ into account in the $\mathrm{He}^{+}$ionization cross section (PTra $\alpha \sigma_{\text {eff }} \mathrm{LI}$ ); and (v) $\sigma_{\text {eff }}$, multiplying the electron-capture cross sections by a factor of two (PTra $\alpha \sigma_{\text {eff }}$ F2). (b) Ratio of the measured $M_{1, \exp }$ to simulated values $M_{1, \text { sim }}$ using (i) ( $\alpha$ fitted Rudd), (ii) ( $\alpha$ Barkas), (iii) $\left(\alpha \sigma_{\text {eff }}\right)$, and (v) (PTra $\alpha \sigma_{\text {eff }}$ F2). The statistical uncertainties of the simulations are contained within the symbols of the graphs. 
For alpha particles, $M_{1}$ values calculated using the fitted Rudd model also agree with the measured data (within experimental uncertainties) for energies above $2 \mathrm{MeV}$ (labeled "PTra $\alpha$ fitted Rudd" in the figure). For lower energies, the simulations yield cluster sizes up to about $42 \%$ larger. The maximum value (at an energy of about $400 \mathrm{keV}$ ) is found at a lower energy than the experimental maximum (at $500 \mathrm{keV}$ ).

The Barkas model [56] leads to an underestimation of the $M_{1}$ values across the entire energy range by $10 \%$ to $20 \%$ and as much as $45 \%$ at the lowest investigated energy of about $130 \mathrm{keV}$ ("PTra $\alpha$ Barkas"). As for nitrogen, the Barkas model (using $a=125$ ) appears, therefore, unsuitable as a model for the effective charge of the ionization cross section of propane in track structure simulations.

A much better agreement with the measured data is achieved when using the effective ionization cross section ("PTra $\alpha \sigma_{\text {eff }}$ "). In this case, the maximum value of $M_{1}$ is calculated at about $700 \mathrm{keV}$. For energies above $700 \mathrm{keV}$, the simulated data agree well with the measured data (within the experimental uncertainties), while below this energy, the calculated $M_{1}$ values are up to $24 \%$ smaller. This deviation is large compared to the contribution of electron-loss processes, resulting in a relatively minor reduction of $M_{1}$ values (see Sec. V A1). This justifies the implementation of $\sigma_{\text {eff }}$ of propane instead of the detailed inclusion of charge-transfer interactions in the simulation, thereby neglecting the further transport of electrons produced in electron-loss processes.

Considering the contribution of electron-loss ionization processes in the $\mathrm{He}^{+}$ionization cross section (see Sec. IV G) reduces the mean ionization cluster size by as much as $7 \%$ in the energy region between $200 \mathrm{keV}$ and $1 \mathrm{MeV}$ (labeled "PTra $\alpha \sigma_{\text {eff }}$ LI" in Fig. 13). The influence on the propane data is significant and larger than for nitrogen. This is because the $\mathrm{He}^{+}$ionization cross section used for propane is larger than the charge-transfer cross sections.

Another unknown bias of propane cross sections may arise from the sparse and inconsistent literature data on charge-transfer cross sections of propane (see Secs. IV F2 and IV G). The review conducted in this work indicated that propane cross sections may be a factor of two larger than those of methane. This factor is considered in the calculation of the mean ionization cluster size ("PTra $\alpha \sigma_{\text {eff }}$ F2"). For energies below $1 \mathrm{MeV}$, the $M_{1}$ values are then as much as $6 \%$ larger than those calculated previously (i.e., "PTra $\alpha \sigma_{\text {eff }}$ "). $M_{1}$ values calculated by this approach are then up to $18 \%$ lower than the experimental data, yielding the best agreement of all simulation results.

\section{B. Ion energy loss}

Another quantity used for the benchmark test of PTRA is the energy loss of incident protons and alpha particles during the passage of more than $30 \mathrm{~cm}$ of gas in the nanodosimeter setup. For this purpose, the average energy of projectile ions arriving at the trigger detector were measured and calculated. Results from the simulations are also compared to the energy loss calculated for the same geometry but using the stopping power from the PSTAR and ASTAR libraries [61].

For protons in nitrogen, the energy losses determined using the three approaches deviate by less than $3 \%$, which

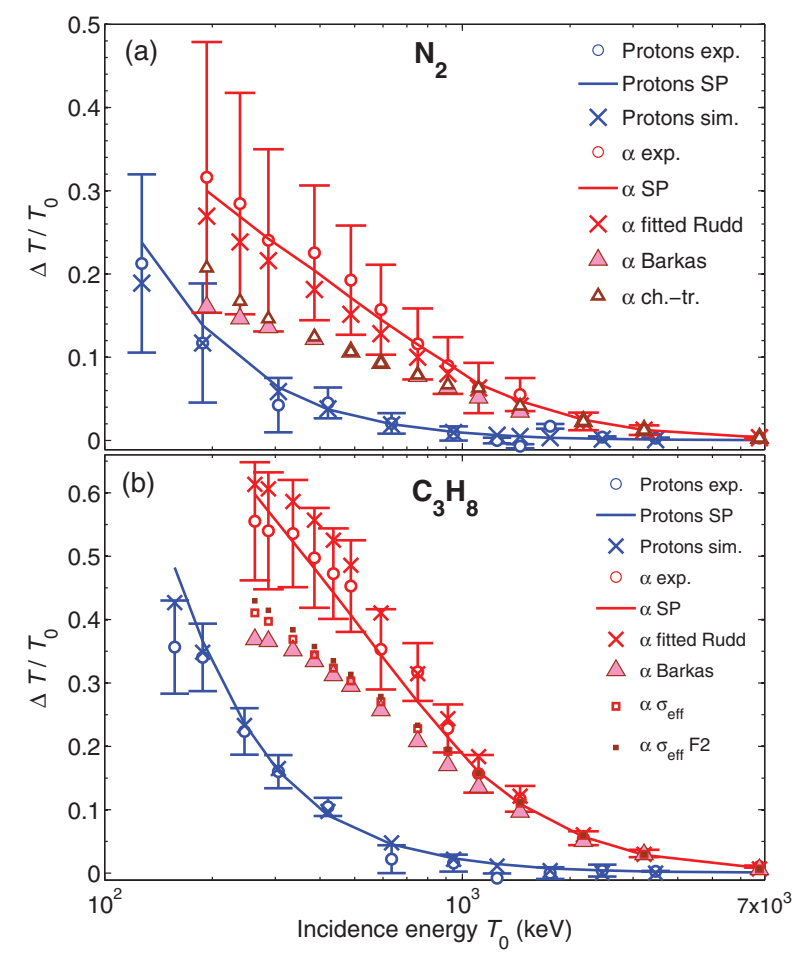

FIG. 14. (Color online) Relative energy loss of incident protons and alpha particles, obtained from measurements (exp), the stopping power (SP), and simulations ( $\mathrm{sim}$ ) in (a) nitrogen and (b) propane after passing through the nanodosimeter volume. Different cross section models for alpha particles were used [(i), (ii), and (iii) in captions of Figs. 12 and 13, as well as (v) in the latter caption].

is well within the experimental uncertainty of as much as $11 \%$ [Fig. 14(a)]. The assessment of the uncertainty in energy loss was based on the uncertainty of the experimental energy determination at the trigger detector. Experimental values of the energy loss of alpha particles agree well (within 2\%) with data calculated from the stopping power for the entire energy range investigated. Also, simulations of alpha particles in nitrogen using the fitted Rudd model led to less than $5 \%$ deviation in energy loss. Deviations of up to $12 \%$ and $16 \%$ between measured and simulated energies are found at energies below $1 \mathrm{MeV}$ when effective ionization cross sections or the Barkas model were used, respectively. For all investigated cross section models, this disagreement is, for most data points, within the experimental uncertainties evaluated for the energy loss (which increased with decreasing energy to as much as $17 \%$ ).

For propane, good agreement between the energy loss of protons determined from experiment, simulations, and stopping power can be seen in Fig. 14(b). Here, the deviation in energy losses is less than 3\%, with the exception of the lowest initial energy of $157 \mathrm{keV}$, which was reduced to $100 \mathrm{keV}$ in the experiment but to only $87 \mathrm{keV}$ in the simulations. Using the stopping power to calculate the energy at the detector position gives $81 \mathrm{keV}$, which is closer to the simulated value.

The simulated energy loss of alpha particles with energies above $1 \mathrm{MeV}$ in propane (obtained by the different cross section models) compares within $5 \%$ with the measured values. Below $1 \mathrm{MeV}$, the calculated energy loss of the alpha particles 
shows significant deviations from the measured values for some of the investigated cross section models. The experimental values, on the other hand, are supported by the stopping power calculations from which they deviate by no more than $5 \%$ (this is within experimental uncertainties determined for the energy loss, which increased with decreasing energy to as much as $8 \%$ ). Similar to the simulation results for nitrogen, the use of the fitted Rudd model in the simulations leads to as much as $7 \%$ higher energy loss than in the experiment, while the application of the Barkas model or the effective ionization cross section resulted in up to $19 \%$ or $15 \%$ lower energy loss when compared to the measured data. Multiplying the charge-transfer cross sections of propane by a factor of two leads to calculated energy losses of as much as $13 \%$ lower than the measured data [labeled "PTra $\alpha \sigma_{\text {eff }}$ F2" in Fig. 14(b)]. The energy losses for alpha particles of incident energy below $1 \mathrm{MeV}$ are, nonetheless, significantly lower (experimental uncertainty is up to $10 \%$ ) when charge-transfer processes were considered in the simulations.

\section{Contribution of secondary electrons to $M_{1}$}

Secondary electrons are produced within the sensitive volume as well as in the surrounding medium by ionization and electron-loss processes of the projectiles. The contribution of secondary electrons to $M_{1}$ was investigated to gain insight into the importance of the ionization cross sections of electrons for the central passage of a primary ion beam through a sensitive volume.

Figure 15 shows the fraction of $M_{1}$ that is produced by secondary electrons only (instancing results in nitrogen). It can be seen that the contribution of secondary electrons to $M_{1}$ is generally smaller than that of ions, but still significant (between about $15 \%$ and $30 \%$ for the range of ion energies investigated in this work). This is due to the fact that ions of such energies traverse the sensitive volume without being scattered, whereas electrons experience a change in direction due to elastic and inelastic scattering. Electrons therefore have a probability of being scattered out of the sensitive volume before undergoing

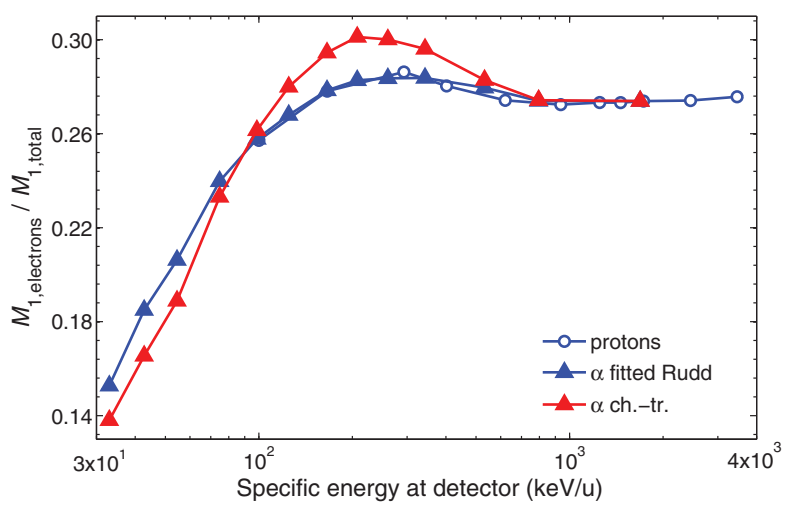

FIG. 15. (Color online) Ratio of the secondary electron contribution to the simulated mean ionization cluster size $\left(M_{1, \text { electrons }}\right)$ to the total mean ionization cluster size $\left(M_{1, \text { total }}\right)$ for the passage of protons and helium particles through the center of the sensitive volume of the nanodosimeter operated with nitrogen. For helium particles, either the fitted Rudd model or charge-transfer cross sections were used in the code. ionization. This is particularly the case for electrons with energies above $400 \mathrm{eV}$, since their ionization mean free path is larger than the diameter of the sensitive volume.

In the overlapping energy range, the contribution from secondary electrons to $M_{1}$ is similar for protons and alpha particles (fitted Rudd model), since the electron spectra produced by protons and alpha particles of equal velocity are the same. In both cases, the electron contribution increases with increasing energy to about $28 \%$ at $200 \mathrm{keV}$, then plateaus at higher energies. The electron contribution was also assessed when accounting for charge transfer, where the electron contribution to the $M_{1}$ produced by helium particles of energies below about $100 \mathrm{keV} / \mathrm{u}$ drops by about $2 \%$ due to the inclusion of electron capture in $M_{1}$. This is in agreement with the differences observed in $M_{1}$, determined from the same kind of simulations with and without including electron-capture events to $M_{1}$. For energies between $100 \mathrm{keV} / \mathrm{u}$ and $1 \mathrm{MeV} / \mathrm{u}$, the electron contribution is by as much as $2 \%$ larger than the one obtained by the fitted Rudd model due to secondary electrons produced in electron-loss processes.

\section{CONCLUSIONS}

Cross section data for ion and electron scattering in gases are required for modeling the track structure of radiation. The validity of these data is essential for the accurate calculation of transport parameters and ionization yields with applications in detector development, space research, and radiation dosimetry. Unfortunately, those data are scarce and often discrepant in the literature; ionization cross section data for the interaction of light ions in propane were, for example, not found. Propane cross section data were therefore derived from those of methane. The literature review in this work contributes to the collection and dissemination of ionization and charge-transfer cross section data for electron, proton, and helium particle interactions with nitrogen, methane, and propane. Those data were obtained from measurements or theoretical calculations or described by semiempirical models. In this work, data for ion projectiles of energies between $100 \mathrm{keV}$ and $20 \mathrm{MeV}$ were of interest. The simulation of proton and helium particle transport in the PTB/WIS nanodosimeter, using the PTRA Monte Carlo track structure code, successfully benchmarked a set of cross section data of nitrogen and propane by comparing the calculated energy loss and mean ionization cluster size $M_{1}$ to measured results. This benchmark test offered the possibility to investigate the influence of different models and subsequent parametrizations of ionization cross sections on the calculated quantities. This influence was significant and the observed deviations may be used as an estimate of the expected overall uncertainty of calculated results, arising from uncertainties of the cross section data.

Ionization cross sections of nitrogen and methane for protons in the energy range of interest in this work, are mostly in good agreement within the experimental uncertainties. Those data are well described by the semiempirical Rudd and Green models but the Green model significantly overestimates the cross section data for protons and those based on electronimpact data at high energies $(30 \%$ at $10 \mathrm{keV})$. The use of the Rudd model [28] in simulations with both nitrogen and propane led to a good agreement (within the experimental 
uncertainties) with the measured energy losses as well as with the $M_{1}$, except for proton energies below $300 \mathrm{keV}$ in nitrogen, where measured and simulated $M_{1}$ values deviated by up to $17 \%$. A comparison of this deviation to the uncertainties associated with the experimental cross section data (between $10 \%$ and $25 \%$ ) leads to the conclusion that the benchmark test for protons was successful. Therefore, the Rudd model is recommended as an interpolation function for proton-impactionization cross section data.

Impact-ionization cross sections of nitrogen for $\mathrm{He}^{2+}$ and $\mathrm{He}^{0}$ have been measured by few authors, but are in agreement within the experimental uncertainties (up to $17 \%$ deviation). For $\mathrm{He}^{+}$, larger discrepancies (up to $25 \%$ ) occur between the reported data, which were in all cases obtained by subtracting the measured electron-loss cross section $\sigma_{12}$ from the cross section for the production of electrons $\sigma_{-}$. For methane, only one author reported ionization cross sections for $\mathrm{He}^{2+}$ and $\mathrm{He}^{+}$ions. The best fit curves for alpha particles was obtained by refitting the Rudd model [28] (originally for protons) to the experimental cross section data, while Gaussian functions were used to fit the data for $\mathrm{He}^{+}$and $\mathrm{He}^{0}$ projectiles. Charge-transfer cross sections were measured by several groups but often in different energy ranges. The data in overlapping energy ranges agree mostly within $20 \%$. Two data sets, however, show deviations of more than a factor of two which seem to be of systematic origin. Model functions were fitted to the charge-transfer data of nitrogen and also used for those of methane due to the similarity of the charge-transfer cross sections of both molecules. Equilibrium fractions of the charge states in a helium particle beam, calculated using the model functions of the charge-transfer cross sections deviate by up to $20 \%$ from those measured by two groups in complementing energy ranges. The measured equilibrium fractions were also fitted by model functions for use in the Monte Carlo code. Based on the data for equilibrium fractions and charge-transfer cross sections, effective ionization cross sections, accounting also for ionization by electron-capture processes, were calculated.

The different interpolation model functions were used in the code PTRA to parametrize ionization and charge-transfer cross sections of incident alpha particles. Results for the $M_{1}$ values and the energy loss of incident alpha particles are consistent within the simulated data since a larger energy loss corresponds to the larger $M_{1}$ values, obtained when using only the fitted Rudd model and compared to simulations including charge-transfer processes. This is due to the role played by charge-transfer processes: When the $\mathrm{He}^{2+}$ ion undergoes electron capture, the helium particles does not experience (significant) energy loss, but it has a lower charge state $\left(\mathrm{He}^{+}\right.$ or $\mathrm{He}^{0}$ ) and, consequently, a lower ionization cross section. Therefore, the average ionization cross section of the helium particle is reduced when charge transfer is considered in the simulations. For this reason, helium particles simulated under these conditions lose less energy when traversing the gas than those simulated using the fitted Rudd model. On the other hand, the discrepancy of the calculated energy loss to the experimental values is larger when accounting for charge transfer (up to $13 \%$ lower energy loss) instead of using the fitted Rudd model for alpha particle ionization cross sections (up to $7 \%$ higher). This is in contradiction with the larger deviation of the calculated $M_{1}$ values from the experimental data when the fitted Rudd model is used (up to $42 \%$ larger and $24 \%$ lower $M_{1}$ when the fitted Rudd model or the charge-transfer data are used, respectively). A possible reason for this contradiction is that the calculated energy loss of an ion per single interaction is not large enough (the HKS model was used in PTRA [62]). If this would be the case, the calculated total energy loss would increase (particularly for particles of lower energies), while the $M_{1}$ values would not significantly change. Further investigation of the cross section data used to determine the energy loss per interaction in PTRA is therefore necessary and will be conducted.

Furthermore, the results of this work lead to the conclusion that the Barkas model [56] is inadequate for use in track structure simulations as the largest deviations (up to 65\%) of all calculated data to the experimental values are observed. It can, however, not be disclaimed that the low energy loss could be due to an underestimated energy loss per single interaction (as described above), while lower $M_{1}$ values definitely also arise from the neglected contribution of ionizations by electron capture (the contribution of those ionizations to $M_{1}$ at energies below $1 \mathrm{MeV}$ is up to $16 \%$ ). The latter reason alone, in fact, leads to the unsuitability of the Barkas model for a simulation of nanodosimetric quantities. However, also the discrepancy observed in Sec. IV G has to be further investigated. Here it was shown that the effective ionization cross section based on the Barkas model was only 1/3 of the same cross section, calculated from charge-transfer data and equilibrium charge states of helium particles.

The overall contribution of ionizations by secondary electrons to $M_{1}$ was found to be as much as $30 \%$ for a central passage of an ion beam through the sensitive volume. This underlines the importance of accurate electron-impact cross section data, which deviated by up to $65 \%$ and $30 \%$ for propane and nitrogen, respectively. Particulary in the view that electrons are exclusively responsible for energy depositions in the vicinity of an ion trajectory, more accurate cross section data and benchmark experiments for such a scenario are needed [63].

Based on the literature review and the results in this work, the BEB model and Chouki model are recommended as interpolation functions for electron-impact-ionization cross sections of nitrogen and propane, respectively. For protonimpact, the Rudd model [28] is recommended. Due to the best agreement of calculated and measured $M_{1}$ and energy losses, the implementation of the charge-transfer processes as used in (v1) (Sec. IV E) is recommended when alpha particles are incident. For this purpose, the fitted Rudd model and Gaussian functions are proposed as interpolation models for ionization cross sections for $\mathrm{He}^{2+}, \mathrm{He}^{+}$, and $\mathrm{He}^{0}$, where $\mathrm{He}^{0}$ cross section of nitrogen may also be used for methane. For propane, the methane data should be multiplied by a factor of 2.9. Model functions for the charge-transfer cross sections in nitrogen are also proposed and may be multiplied by a factor of two to obtain a reasonable estimate for the data of propane. Alternatively to the calculation of the specific charge state of each helium projectile in every simulation step, an effective ionization cross section is proposed in Eq. (7). In this case, the production of electrons in electron-loss processes and their subsequent potential to produce additional ionizations is 
neglected in favor of computation time. Ionizations by those neglected electrons have, however, only a minor effect (as much as $7 \%$ ) on the simulation results.

The uncertainty of calculated results for $M_{1}$ and energy loss arising from different parametrizations of effective ionization cross sections may be estimated from the difference between the values determined in "PTra $\alpha$ ch.-tr." and those from "PTra $\alpha$ v3" for nitrogen. Both data sets, as well as the one used to calculate "PTra $\alpha$ ch.-tr. LI," seem equally justified to model a realistic effective ionization cross section. In this case, the calculated $M_{1}$ values would have an assigned uncertainty of up to $20 \%$ for helium projectile energies between $300 \mathrm{keV}$ and $1.5 \mathrm{MeV}$ and an uncertainty below $2 \%$ for energies outside this range. The energy loss, on the other hand, is less sensitive to the different parametrizations of the effective ionization cross section, showing deviations below $2 \%$.

The PTRA code using the recommended cross section data of nitrogen and propane can be considered as being successfully benchmarked by the experiments with protons and alpha particles of energies between $100 \mathrm{keV}$ and $20 \mathrm{MeV}$ in the nanodosimeter. Further improvement is necessary, and requires the measurement of cross section data, particularly for light ions in propane but also electron and ion cross section data of nitrogen and methane are needed. This work will be continued for differential cross section data as well as for excitation and elastic scattering cross sections to further improve particle transport simulations with the code PTRA.

\section{ACKNOWLEDGMENTS}

We thank Daniel Bennett for carefully reading the manuscript. The research leading to these results was, in part, carried out within the EMRP joint research Project No. SIB06 BioQuaRT. The EMRP is jointly funded by the EMRP participating countries within EURAMET and the European Union.

\section{APPENDIX A: BEB MODEL}

The BEB model [19] describes the electron-impactionization cross section for the electron energy range up to about $10 \mathrm{keV}$, without requiring empirical parameters. It combines the binary-encounter theory with the Bethe-Born approximation, which are expected to be realistic models for low and high incidence electron energies, respectively. The Bethe-Born approximation is applicable if the energy of the incident electron is much greater than that of the bound target electrons. In this case, the projectile potential can be treated as a small perturbation to the target electrons because energy transfers, produced in soft collisions, are likely to be small. In the binary-encounter theory, a velocity distribution is assigned to the bound target electrons, enabling a better description of hard collisions. The total ionization cross section is described by the (nonrelativistic) BEB model as

$$
\begin{aligned}
\sigma_{\text {ion }}(T)= & \sum_{i=1}^{i_{\max }}\left(\frac { 4 \pi a _ { 0 } ^ { 2 } R ^ { 2 } } { t + u + 1 } \frac { N _ { i } } { B _ { i } ^ { 2 } } \left\{0.5\left(1-\frac{1}{t^{2}}\right) \ln (t)\right.\right. \\
& \left.\left.+\left[\left(1-\frac{1}{t^{2}}\right)-\frac{\ln (t)}{t+1}\right]\right\}\right),
\end{aligned}
$$

where $R$ is the Rydberg constant, $a_{0}$ is the Bohr radius, $i$ is an index for the subshells, and $N_{i}$ is the number of electrons
TABLE VI. Parameters of the BEB model for nitrogen [18].

\begin{tabular}{cccc}
\hline \hline$i$ & $N_{i}$ & $B_{i}(\mathrm{eV})$ & $U_{i}(\mathrm{eV})$ \\
\hline 1 & 2 & 15.58 & 54.91 \\
2 & 4 & 17.07 & 44.30 \\
3 & 2 & 21.00 & 63.18 \\
4 & 2 & 41.72 & 71.13 \\
\hline \hline
\end{tabular}

occupying the $i$ th shell [18]. The primary electron energy $T$ and the kinetic energy $U_{i}$ of an orbital electron in shell $i$ are normalized to the binding energy of the orbital electron $B_{i}$ to give $t=T / B_{i}$ and $u=U_{i} / B_{i}$. The molecular orbital data for nitrogen are shown in Table VI.

\section{APPENDIX B: CHOUKI MODEL}

Chouki et al. [27] developed a semiempirical model for electron-impact-ionization cross sections of hydrocarbons. Their model is based on the Bethe-Born approximation and includes corrections for the low-energy region, obtained by fitting experimental data. The Chouki model is defined by

$$
\sigma_{\text {ion }}(T)=4 \pi a_{0}^{2} R \frac{C}{T} \ln \left(1+\frac{T-I}{R}\right) \Phi(T),
$$

with

$$
\Phi(T)=a_{1} e^{-b_{1} / T}+a_{2} e^{-b_{2} / T^{2}}+a_{3} e^{-b_{3}(T-I) / T^{2}},
$$

where $R$ is the Rydberg constant, $a_{0}$ is the Bohr radius, and $I$ is the ionization threshold of $11.08 \mathrm{eV}$ for propane. The parameters for propane were obtained by fitting the model to the average value of experimental ionization cross sections in literature (see Sec. IV B), giving $C=16.0, a_{1}=1.0$, $a_{2}=-59.96, a_{3}=59.84, b_{1}=45.84 \mathrm{eV}, b_{2}=0.16(\mathrm{eV})^{2}$, and $b_{3}=-0.13 \mathrm{eV}$.

\section{APPENDIX C: RUDD MODEL}

Rudd et al. [28] fitted a semiempirical function to experimental proton ionization cross sections of various gases which they comprehensively collected from the literature and critically evaluated by assigning a weight to each data set. This function consists of two components, appropriate for the high- and low-energy regions ( $\sigma_{\text {high }}$ and $\sigma_{\text {low }}$, respectively). The Rudd model function for protons of energy $T$ is given by

$$
\begin{aligned}
\sigma_{-}(T) & =\left(\sigma_{\text {low }}^{-1}+\sigma_{\text {high }}^{-1}\right)^{-1}, \\
\sigma_{\text {low }} & =4 \pi a_{0}^{2} C_{\text {Rudd }} x^{D_{\text {Rudd }}}, \\
\sigma_{\text {high }} & =4 \pi a_{0}^{2}\left[A_{\text {Rudd }} \ln (1+x)+B_{\text {Rudd }}\right] / x,
\end{aligned}
$$

TABLE VII. Parameters of the Rudd model for protons [28] and alpha particles in nitrogen and methane.

\begin{tabular}{lcccc}
\hline \hline & $\mathrm{N}_{2}, \mathrm{H}^{+}$ & $\mathrm{N}_{2}, \mathrm{He}^{2+}$ & $\mathrm{CH}_{4}, \mathrm{H}^{+}$ & $\mathrm{CH}_{4}, \mathrm{He}^{2+}$ \\
\hline$A_{\text {Rudd }}$ & 3.82 & 3.82 & 4.55 & 4.55 \\
$B_{\text {Rudd }}$ & 2.78 & 2.78 & 2.07 & 2.07 \\
$C_{\text {Rudd }}$ & 1.80 & 0.85 & 2.54 & 1.15 \\
$D_{\text {Rudd }}$ & 0.70 & 0.70 & 1.08 & 1.08 \\
\hline \hline
\end{tabular}


TABLE VIII. Parameters of the model function for the ionization cross sections [Eq. (E1)].

\begin{tabular}{lccc}
\hline \hline & $\mathrm{N}_{2}, \sigma_{\mathrm{He}^{+}}$ & $\mathrm{N}_{2}, \sigma_{\mathrm{He}^{0}}$ & $\mathrm{CH}_{4}, \sigma_{\mathrm{He}^{+}}$ \\
\hline$a_{1}$ & 6.90 & 8.00 & 7.60 \\
$b_{1}$ & 2.30 & 2.11 & 2.30 \\
$c_{1}$ & 1.70 & 1.26 & 1.60 \\
$a_{2}$ & 2.62 & & 3.90 \\
$b_{2}$ & 2.43 & & 2.53 \\
$c_{2}$ & 0.70 & & 0.60 \\
\hline \hline
\end{tabular}

where $R$ is the Rydberg constant, $a_{0}$ the Bohr radius, and $x=T / R$. The fit parameters determined by Rudd et al. for the impact of protons on nitrogen and methane are provided in Table VII, together with those for the impact of alpha particles (obtained by fitting the Rudd model to measured cross sections of $\mathrm{N}_{2}$ and $\mathrm{CH}_{4}$ ).

\section{APPENDIX D: GREEN MODEL}

The model proposed by Green and McNeal [34] for the ionization cross section of protons is a semiempirical analytic function, which has been fitted to experimental proton ionization cross sections of various rare gases and diatomic molecules. In the case of $\mathrm{N}_{2}$, they used the data of de Heer et al. [30] in the low-energy range and for high energies the model data of Hooper et al. [35], which is based on the Bethe-Born approximation and has been fitted to experimental data itself. by

The Green model for protons of energy $T$ (in $\mathrm{keV}$ ) is given

$$
\sigma_{\text {ion }}(T)=\sigma_{0} \frac{(Z a)^{\Omega}(T-I)^{v}}{J^{\Omega+v}+T^{\Omega+v}},
$$

with the parameters $\sigma_{0}=2.93 \times 10^{-16} \mathrm{~cm}^{2}$, the total number of electrons in the target $Z=7, a=120.36, \Omega=0.75, v=$ $0.77, J=67.15$, and the ionization threshold $I=15.58 \mathrm{eV}$.

\section{APPENDIX E: PHENOMENOLOGICAL MODELS FOR IONIZATION AND CHARGE-TRANSFER CROSS SECTIONS}

The experimental data for ionization and charge-transfer cross sections as well as for equilibrium charge states of helium
TABLE IX. Parameters of the model functions for charge-transfer cross sections [Eqs. (E2) and (E3)].

\begin{tabular}{lcrcrrr}
\hline \hline & $\sigma_{21}$ & $\sigma_{20}$ & $\sigma_{10}$ & $\sigma_{12}$ & $\sigma_{02}$ & $\sigma_{01}$ \\
\hline$p_{1}$ & 15.62 & 17.51 & 15.55 & & & \\
$q_{1}$ & -0.03 & -0.11 & -0.11 & & & \\
$p_{2}$ & 0.012 & 0.10 & 0.584 & & & \\
$q_{2}$ & 1.71 & 1.33 & 0.78 & & & \\
$r_{1}$ & & & & -0.83 & -1.19 & -0.38 \\
$r_{2}$ & & & & 5.00 & 6.88 & 1.93 \\
$r_{3}$ & & & & -23.55 & -26.19 & -17.82 \\
\hline \hline
\end{tabular}

projectiles were fitted as function of $x=\log _{10}(T / \mathrm{keV})$, where $T$ is the projectile energy. The best fit curves, valid in the energy range between $100 \mathrm{keV}$ and $20 \mathrm{MeV}$, are shown in Secs. IVE and IVF). Parameter values are provided in Table VIII.

The model functions for $\mathrm{He}^{+}$and $\mathrm{He}^{0}$ were obtained by the Gaussian expressions

$$
\sigma_{\mathrm{He}^{z}}(T)=\left[a_{1} e^{-\left(\frac{x-b_{1}}{c_{1}}\right)^{2}}+a_{2} e^{-\left(\frac{x-b_{2}}{c_{2}}\right)^{2}}\right] 10^{-16} \mathrm{~cm}^{2}
$$

for helium particles of charge $Z$.

The electron-capture cross sections of nitrogen are described by

$$
\log _{10}\left(\sigma_{21} / \mathrm{cm}^{2}\right)=-p_{1} e^{q_{1} x}-p_{2} e^{q_{2} x}
$$

and the model functions for nitrogen electron-loss cross sections are

$$
\log _{10}\left(\sigma_{12} / \mathrm{cm}^{2}\right)=r_{1} x^{2}+r_{2} x+r_{3}
$$

Parameters for both Eqs. (E2) and (E3) are given in Table IX.

The best-fit model functions obtained for the equilibrium fractions of helium charge states measured by Barnett and Stier as well as Pivovar et al. [48,53] are obtained by

$$
\begin{aligned}
& f_{0, \exp }=1308 /\left(35.22+e^{0.62 x^{2}}\right)^{2}, \\
& f_{2, \exp }=1.045 \times 10^{-7} /\left(1.044 \times 10^{-7}+e^{-5.6 x}\right), \\
& f_{1, \exp }=1-f_{2, \exp }-f_{0, \exp .}
\end{aligned}
$$

[1] H. H. Rossi and M. Zaider, Microdosimetry and Its Applications (Springer-Verlag, Heidelberg, 1996).

[2] A. M. Kellerer, Radiat. Prot. Dosim. 99, 17 (2002).

[3] W. Friedland, P. Jacob, H. G. Paretzke, and T. Stork, Radiat. Res. 150, 170 (1998).

[4] B. Grosswendt and S. Pszona, Radiat. Environ. Biophys. 41, 91 (2002).

[5] H. Nikjoo, P. O’Neill, M. Terrissol, and D. T. Goodhead, Radiat. Environ. Biophys. 38, 31 (1999).

[6] R. W. Schulte, A. J. Wroe, V. A. Bashkirov, G. Y. Garty, A. Breskin, R. Chechik, S. Shchemelinin, E. Gargioni, B. Grosswendt, and A. B. Rosenfeld, Z. Med. Phys. 18, 286 (2008).
[7] G. Garty, S. Shchemelinin, A. Breskin, R. Chechik, G. Assaf, I. Orion, V. Bashkirov, R. Schulte, and B. Grosswendt, Nucl. Instr. Meth. Phys. Res. A 492, 212 (2002).

[8] B. Grosswendt, Radiat. Environ. Biophys. 41, 103 (2002).

[9] H. Nikjoo, S. Uehara, D. Emfietzoglou, and F. A. Cucinotta, Radiat. Meas. 41, 1052 (2006).

[10] G. Hilgers, E. Gargioni, B. Grosswendt, and S. Shchemelinin, Radiat. Prot. Dosim. 126, 467 (2007).

[11] H. Bethe, Ann. Phys. 397, 325 (1930).

[12] M. Inokuti, Rev. Mod. Phys. 43, 297 (1971).

[13] B. Grosswendt and W. Y. Baek, in Basic Physical Data in Organic Gases (INFN-Laboratori Nazionali di Legnaro, Padova, Italy, 2000), pp. 5-26. 
[14] N. Stolterfoht, R. D. DuBois, and R. D. Rivarola, Electron Emission in Heavy Ion-atom Collisions (Springer, Berlin, 1997).

[15] L. J. Puckett, G. O. Taylor, and D. W. Martin, Phys. Rev. 178, 271 (1969).

[16] Y. Itikawa, J. Phys. Chem. Ref. Data 35, 31 (2006).

[17] D. Rapp and P. Englander-Golden, J. Chem. Phys. 43, 1464 (1965).

[18] W. Hwang, Y.-K. Kim, and M. E. Rudd, J. Chem. Phys. 104, 2956 (1996).

[19] Y.-K. Kim and M. E. Rudd, Phys. Rev. A 50, 3954 (1994).

[20] National Institute of Standards and Technology, Electronimpact Cross Sections for Ionization and Excitation (2012), http://physics.nist.gov/PhysRefData/Ionization/molTable.html.

[21] N. Durić, I. Čadež, and M. Kurepa, Int. J. Mass. Spectrom. 108, R1 (1991).

[22] B. L. Schram, M. J. van der Wiel, F. J. de Heer, and H. R. Moustafa, J. Chem. Phys. 44, 49 (1966).

[23] H. Nishimura and H. Tawara, J. Phys. B 27, 2063 (1994).

[24] V. Grill, G. Walder, D. Margreiter, T. Rauth, H. U. Poll, P. Scheier, and T. D. Märk, Z. Phys. D 25, 217 (1993).

[25] G. L. C. de Souza, M.-T. Lee, I. P. Sanches, P. Rawat, I. Iga, A. S. dos Santos, L. E. Machado, R. T. Sugohara, L. M. Brescansin, M. G. P. Homem et al., Phys. Rev. A 82, 012709 (2010).

[26] M. Vinodkumar, K. N. Joshipura, C. G. Limbachiya, and B. K. Antony, Eur. Phys. J. D 37, 67 (2006).

[27] A. Chouki, Ph.D. thesis, University Paul Sabatier, Toulouse, 1994.

[28] M. E. Rudd, Y.-K. Kim, D. H. Madison, and J. W. Gallagher, Rev. Mod. Phys. 57, 965 (1985).

[29] J. B. Crooks and M. E. Rudd, Phys. Rev. A 3, 1628 (1971).

[30] F. J. de Heer, J. Schutten, and H. Moustafa, Physica 32, 1766 (1966).

[31] H. Knudsen, U. Mikkelsen, K. Paludan, K. Kirsebom, S. P. Møller, E. Uggerh $\varnothing$ j, J. Slevin, M. Charlton, and E. Morenzoni, J. Phys. B 28, 3569 (1995).

[32] M. E. Rudd, R. D. DuBois, L. H. Toburen, C. A. Ratcliffe, and T. V. Goffe, Phys. Rev. A 28, 3244 (1983).

[33] S. K. Allison, Rev. Mod. Phys. 30, 1137 (1958).

[34] A. E. S. Green and R. J. McNeal, J. Geophys. Res. 76, 133 (1971).

[35] J. W. Hooper, D. S. Harmer, D. W. Martin, and E. W. McDaniel, Phys. Rev. 125, 2000 (1962).

[36] I. Ben-Itzhak, K. D. Carnes, D. T. Johnson, P. J. Norris, and O. L. Weaver, Phys. Rev. A 49, 881 (1994).

[37] H. Luna, E. G. Cavalcanti, J. Nickles, G. M. Sigaud, and E. C. Montenegro, J. Phys. B 36, 4717 (2003).
[38] D. J. Lynch, L. H. Toburen, and W. E. Wilson, J. Chem. Phys. 64, 2616 (1976).

[39] W. E. Wilson and L. H. Toburen, Phys. Rev. A 11, 1303 (1975).

[40] M. E. Rudd, T. V. Goffe, A. Itoh, and R. D. DuBois, Phys. Rev. A 32, 829 (1985).

[41] R. A. Langley, D. W. Martin, D. S. Harmer, J. W. Hooper, and E. W. McDaniel, Phys. Rev. 136, A379 (1964).

[42] L. I. Pivovar, Y. Z. Levchenko, and A. N. Grigor'ev, Sov. Phys. JETP 27, 699 (1968).

[43] M. E. Rudd, T. V. Goffe, and A. Itoh, Phys. Rev. A 32, 2128 (1985).

[44] E. S. Solov'ev, R. N. Il'in, V. A. Oparin, and N. V. Fedorenko, Sov. Phys. JETP 18, 342 (1964).

[45] P. D. Fainstein, V. H. Ponce, and R. D. Rivarola, J. Phys. B 24, 3091 (1991).

[46] G. H. Gillespie, Phys. Lett. A 93, 327 (1983).

[47] A. Itoh, M. Asari, and F. Fukuzawa, J. Phys. Soc. Jpn. 48, 943 (1980).

[48] C. F. Barnett and P. M. Stier, Phys. Rev. 109, 385 (1958).

[49] H. B. Gilbody, K. F. Dunn, R. Browning, and C. J. Latimer, J. Phys. B 4, 800 (1971).

[50] H. B. Gilbody, K. F. Dunn, R. Browning, and C. J. Latimer, J. Phys. B 3, 1105 (1970).

[51] P. Hvelplund and E. Horsdal Pedersen, Phys. Rev. A 9, 2434 (1974).

[52] A. Itoh, K. Ohnishi, and F. Fukuzawa, J. Phys. Soc. Jpn. 49, 1513 (1980).

[53] L. I. Pivovar, V. M. Tubaev, and M. T. Novikov, Sov. Phys. JETP 14, 20 (1962).

[54] S. T. Manson and L. H. Toburen, Phys. Rev. Lett. 46, 529 (1981).

[55] M. Sataka, A. Yagishita, and Y. Nakai, J. Phys. B 23, 1225 (1990).

[56] W. H. Barkas, Nuclear Research Emulsions. I. Techniques and Theory (Academic Press, New York and London, 1963).

[57] H. H. Heckman, B. L. Perkins, W. G. Simon, F. M. Smith, and W. H. Barkas, Phys. Rev. 117, 544 (1960).

[58] G. Kraft, M. Krämer, and M. Scholz, Radiat. Environ. Biophys. 31, 161 (1992).

[59] G. Hilgers, Radiat. Meas. 45, 1228 (2010).

[60] G. Hilgers, M. U. Bug, E. Gargioni, and H. Rabus [Radiat. Prot. Dosim. (to be published)].

[61] National Institute of Standards and Technology, Pstar and Astar Databases, http://www.nist.gov/pml/data/star/index.cfm.

[62] E. Gargioni and B. Grosswendt, Nucl. Instrum. Meth. A 580, 81 (2007).

[63] V. Conte, P. Colautti, L. D. Nardo, A. Ferretti, M. Poggi, D. Moro, M. Lombardi, G. Tornielli, and B. Grosswendt, Radiat. Prot. Dosim. 143, 455 (2011). 